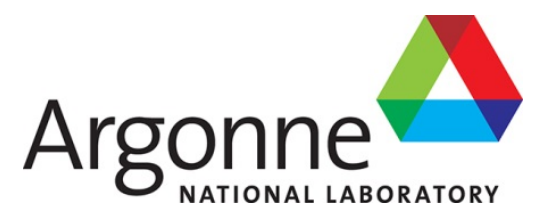

ANL/NSE-20/46

\title{
An Update on Cardinal: Toward Full Core Pebble Simulations
}

Nuclear Science and Engineering Division 


\section{About Argonne National Laboratory}

Argonne is a U.S. Department of Energy laboratory managed by UChicago Argonne, LLC under contract DE-AC02-06CH11357. The Laboratory's main facility is outside Chicago, at 9700 South Cass Avenue, Argonne, Illinois 60439. For information about Argonne and its pioneering science and technology programs, see www.anl.gov.

\section{DOCUMENT AVAILABLITY}

Online Access: U.S. Department of Energy (DOE) reports produced after 1991 and a growing number of pre-1991 documents are available free at OSTI.GOV (http://www.osti.gov/), a service of the US Dept. of Energy's Office of Scientific and Technical Information.

Reports not in digital format may be purchased by the public from the National Technical Information Service (NTIS):

U.S. Department of Commerce

National Technical Information Service

5301 Shawnee Rd

Alexandria, VA 22312

www.ntis.gov

Phone: (800) 553-NTIS (6847) or (703) 605-6000

Fax: (703) 605-6900

Email: orders@ntis.gov

Reports not in digital format are available to DOE and DOE contractors from the Office of Scientific and Technical Information (OSTI):

U.S. Department of Energy

Office of Scientifice and Technical Information

P.O. Box 62

Oak Ridge, TN 37831-0062

www.osti.gov

Phone: (865) 576-8401

Fax: (865) 576-5728

Email: reports@osti.gov

\section{Disclaimer}

This report was prepared as an account of work sponsored by an agency of the United States Government. Neither the United States Government nor any agency thereof, nor UChicago Argonne, LLC, nor any of their employees or officers, makes any warranty, express or implied, or assumes any legal liability or responsibility for the accuracy, completeness, or usefulness of any information, apparatus, product, or process disclosed, or represents that its use would not infringe privately owned rights. Reference herein to any specific commercial product, process, or service by trade name, trademark, manufacturer, or otherwise, does not necessarily constitute or imply its endorsement, recommendation, or favoring by the United States Government or any agency thereof. The views and opinions of document authors expressed herein do not necessarily state or reflect those of the United States Government or any agency thereof, Argonne National Laboratory, or UChicago Argonne, LLC. 


\section{An Update on Cardinal: Toward Full Core Pebble Simulations}

prepared by

Elia Merzari ${ }^{1}$, Derek Gaston ${ }^{4}$, Ronald Rahaman ${ }^{2}$, Patrick Shriwise ${ }^{2}$, Yu-Hsiang Lan ${ }^{3}$, Misun Min $^{3}$, Haomin Yuan ${ }^{1}$, and Dillon Shaver ${ }^{1}$

${ }^{1}$ Nuclear Science and Engineering Division, Argonne National Laboratory

${ }^{2}$ Computational Science Division, Argonne National Laboratory

${ }^{3}$ Mathematics and Computer Science Division, Argonne National Laboratory

${ }^{4}$ Idaho National Laboratory

May 31, 2020 


\section{Contents}

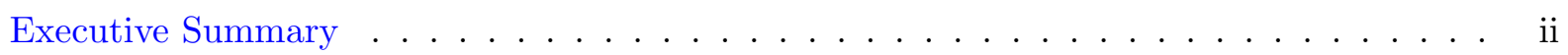

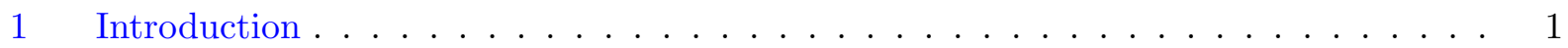

2 Previous work in Cardinal . . . . . . . . . . . . . . . . 3

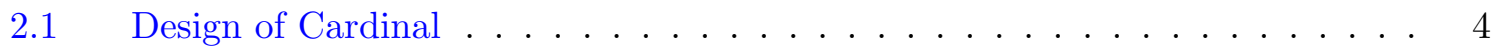

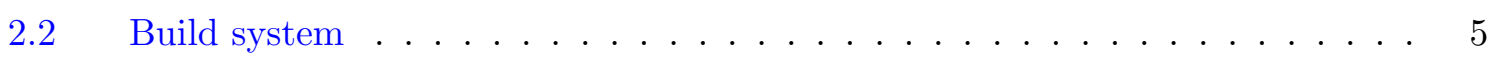

2.3 Verification and validation . . . . . . . . . . . . . 5

2.4 Previous coupled simulations . . . . . . . . . . . . . . . 7

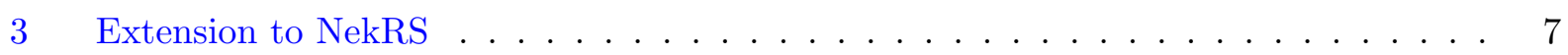

$3.1 \quad$ NekRS . . . . . . . . . . . . . . . . . . . 8

$3.2 \quad$ Updates to Cardinal . . . . . . . . . . . . . . . . . . . . . 11

3.3 Verification . . . . . . . . . . . . . . . . . 13

4 Major update to OpenMC Heat Source . . . . . . . . . . . . . . . . 13

$4.1 \quad$ Updating Temperature Values . . . . . . . . . . . . . . . . . 15

5 Demonstration simulation . . . . . . . . . . . . . . . . . . 15

$5.1 \quad$ Numerical Setup . . . . . . . . . . . . . . . . . . . . . . . . . . . . . . . . . . .

$5.2 \quad$ Results . . . . . . . . . . . . . . . . . . . 19

5.3 Projection to full core . . . . . . . . . . . . . . . . . 21

6 Conclusions and Future Work . . . . . . . . . . . . . . . . . 22

Acknowledgments . . . . . . . . . . . . . . . . . . 23

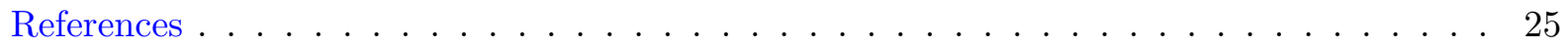




\section{Executive Summary}

The new DOE-NE Center of Excellence for Thermal-fluids applications in Nuclear Energy inaugurated in April 2018 considers and researches novel new solution strategies for historically challenging flow issues that still plague the current fleet of deployed Light Water nuclear Reactors (LWRs) as well as predicting various fluid flow and fluid related issues with advanced reactor technologies. Our advanced thermal-fluids research and development approach synergistically combines three natural, though overlapping, length and time scales in a hierarchal multi-scale approach to avoid the temptation and pitfalls of attempting to develop a single solve-all algorithm for physical fluid flow problems that will span nine orders of magnitude in spatial and temporal scales.

To demonstrate the multi-scale philosophy of the center we focus on Fluoride Cooled High Temperature Reactors (FHRs), and in particular on the Berkley's PB-FHR Mark-I design. The Fluoride salt cooled High temperature Reactor (FHR) is a class of advanced nuclear reactors that combine the robust coated particle fuel form from high temperature gas cooled reactors, direct reactor auxiliary cooling system (DRACS) passive decay removal of liquid metal fast reactors, and the transparent, high volumetric heat capacitance liquid Fluoride salt working fluids - such as Flibe - from molten salt reactors. This combination of fuel and coolant enables FHRs to operate in a high-temperature low-pressure design space that has beneficial safety and economic implications. The PB-FHR reactor relies on a pebble bed approach and pebble bed reactors are in a sense the poster child for multi-scale analysis.

The lower length-scale simulator for pebble reactor cores comprises three physics: neutronics (OpenMC), thermal-fluids (Nek5000/NekRS) and fuel performance (BISON). As part of Center of Excellence ongoing research efforts we have developed Cardinal, a new tool platform for lower length-scale simulation. Cardinal tightly couples all three physics and leverages advances in MOOSE such as the MultiApp system and the concept of MOOSE-wrapped Apps. The present report provides an update on the development of Cardinal with an extension of Cardinal to GPUs. We also perform a first-of-a-kind demonstration simulation on Summit representing a $10 \times$ capability increase in terms of pebble count for Cardinal. 


\section{Introduction}

The DOE-NE Center of Excellence for Thermal-fluids applications in Nuclear Energy inaugurated in April 2018 considers and researches novel new solution strategies for historically challenging issues that still plague the current fleet of deployed Light Water nuclear Reactors (LWR) as well as predicting various fluid flow and fluid related issues with advanced reactor technologies, which includes advanced Small Modular Reactor (SMR) concepts, micro-reactors, and Advanced Reactor Concepts (ARC), utilizing coolants such as liquid metal, chloride and fluoride salts, or gas, for accident-tolerant reactors. These new solution strategies and algorithms will then be implemented into a modern software design methodology, using best software quality practices, and then delivering validated NQA-1 level software to the nuclear power community.

Ultimately, this goal requires a facility where a strong collaborative environment can be established between computational scientists and scientists conducting fluid dynamic experiments. The center addresses a pressing need in advanced reactor development and commercialization. Advanced reactor fluid problems are currently a high priority and lend themselves to advanced modeling and simulation due to the presence of complex flow phenomena and lack of empirical data. In fact, advanced modeling and simulation tools are poised to play an important role: providing deep insight, enhancing the experimental process and accelerating the regulatory process. The center supports a coherent approach, and establishes a true front door for engaging industry. The current approach makes it difficult for customers to engage - single-PI efforts, while individually excellent, have been somewhat disparate, and not coordinated. The CFD/Thermal-Hydraulic Center is truly a multi-lab approach analogous to that used in the very successful fuels M\&S area of NEAMS.

NEAMS and Hub Thermal-Hydraulic/CFD tools are far enough along to warrant this approach. Our advanced thermal-fluids research and development approach synergistically combines three natural, though overlapping, length and time scales in a hierarchal multi-scale approach to avoid the temptation and pitfalls of attempting to develop a single solve-all algorithm for physical fluid flow problems that will span nine orders of magnitude in spatial and temporal scales. A more tractable approach is grouping physics with similar multi-scale requirements into a common algorithm, developing separate software applications to address the separate scales, and then coupling the applications where appropriate. This multi-scale modeling and simulation template has proven to be highly successful in the Nuclear Energy Advanced Modeling and Simulation (NEAMS) program to simulate the evolution of nuclear materials under irradiation. These three overlapping thermal-hydraulic scales are defined across all reactor concepts as:

- Lower Length Scale. The Lower Length Scale will focus upon resolving the high-resolution physics associated with single and multi-phase, highly turbulent conjugate heat transfer (CHT) with highly resolved thermal boundary layers (heat flux).

- Engineering Length scale. The Engineering Length Scale will integrate coarse mesh approaches for homogenized multi-dimensional CHT, such as those found in gas-cooled pebblebed reactors, or three-dimensional sub-channel capabilities tightly coupled to nuclear fuels performance.

- System Scale. System Scale analysis for nuclear reactors is composed of one-dimensional 
fluid flow pipe networks and zero-dimensional system components. These classes of algorithms and corresponding approaches are basically reduced order models (ROM) of the more complex scales and allow for more efficient calculations. These reduced order systems rely heavily on empirical correlations or models, as many of the flow features and phenomena are no longer resolved.

To demonstrate the multi-scale philosophy of the center we focus first on Fluoride Cooled High Temperature Reactors (FHRs), and in particular on Berkley's Pebble Bed Fluoride Salt Cooled High Temperature Reactor (PB-FHR) Mark-1 design. The FHR is a class of advanced nuclear reactors that combine the robust coated particle fuel form from high temperature gas cooled reactors, the direct reactor auxiliary cooling system (DRACS) passive decay heat removal of liquid metal fast reactors, and the neutronically transparent, high volumetric heat capacitance of liquid fluoride salt working fluids, e.g. Flibe, from molten salt reactors. This combination of fuel and coolant enables FHRs to operate in a high-temperature low-pressure design space that has beneficial safety and economic implications. In 2012, UC Berkeley was charged with developing a pre-conceptual design of a commercial prototype FHR - the PB-FHR [1]. The Mark 1 design of the PB-FHR (Mk1 PB-FHR) is a $236 \mathrm{MWt}$ Flibe cooled pebble bed nuclear heat source that drives an open-air Brayton combined-cycle power conversion system. The PB-FHR's pebble bed consists of an enriched uranium fuel core surrounded by an inert graphite pebble region that shields the outer solid graphite region, core barrel and reactor vessel. The fuel reaches an average burnup of $178000 \mathrm{MWt}-\mathrm{d} / \mathrm{MT}$. The Mk1 PB-FHR exhibits strong negative temperature reactivity feedback from the fuel, graphite moderator and the Flibe coolant, but a small positive temperature reactivity feedback of the inner region and from the outer graphite pebble region. Pebble bed reactors are in a sense the prime candidate for the sort of analysis described above.

FHR pebble beds in particular are comprised of hundreds of thousands of pebbles, and a CFD-grade detailed description of the flow field through these pebbles for an entire reactor core is not practical with current simulation technology. However, as this report will demonstrate we have a credible pathway toward a full core high-fidelity CFD capability in this geometry.

For practical, fast-running, design-related purposes porous media formulations are usually employed. However, simple porous media approximations are often incapable of capturing key details of the flow field such as the wall channeling effect due to the change in porosity in proximity of the vessel walls. Advanced formulations for "engineering scale" simulations have the potential to address these issues but data from finer scale simulations is needed to build closure relationships. Pronghorn is the platform of the Center of Excellence for engineering scale thermal-fluids simulations. Finally, finer scale calculations are needed to establish local temperature peaking and fuel temperatures.

An overall multi-physics strategy for an FHR simulation might look like in Figure 1. SAM, the NEAMS tool for systems analysis of advanced reactors with coolants in the liquid phase, drives the simulation of the engineering scale tools (Pronghorn and Rattlesnake/Mammoth). The lower length-scale tools can be run concurrently to provide dynamic closures for the engineering scale or offline to produce correlations (which would be more likely). The lower length-scale simulator may comprise neutronics (e.g., OpenMC), thermal-fluids (Nek5000-NekRS) and fuel performance (BISON). 
Cardinal is the tool developed in the Center of Excellence for lower length-scale simulation. This new platform tightly couples all three physics and leverages advances in MOOSE [2] such as the MultiApp system and the concept of MOOSE-wrapped Apps. Moreover it is designed from the ground-up to scale on massively parallel architectures and perform well on world-class super-computing architectures.

In this report we provide a major update to the high fidelity modeling of FHR pebble beds. A particular result of this year's efforts have been to transition to a hybrid GPU-CPU model to leverage the potential of pre-exascale supercomputer architectures (i.e., Summit).

In particular, we:

- Summarize some of the previous work conducted with Cardinal (section 2);

- Provide an extension of Cardinal to work on GPU-based systems (section 3), this part includes the creation of a new API to NekRS [3]- the new GPU port of Nek5000 - and it includes verification of the coupling to MOOSE;

- Discuss the introduction of mesh-based tallies in OpenMC (section 4) and the introduction of mesh-tally transfer with Cardinal;

- Discuss a set of demo simulations (section 5) related to an FHR comprising 1568 pebbles (10x what previously done with Cardinal), providing a pathway toward full core simulations.

We emphasize the importance of the data generated with these high fidelity conjugate heat transfer simulations of pebble beds. They represent an important stepping stone for developing wall-channeling effect models for Pronghorn, and a resource for confirmatory analysis of reduced order models.

\section{Previous work in Cardinal}

MOOSE was originally developed for solving fully coupled systems of partial differential equations (PDEs) using fully implicit time stepping. To use MOOSE, developers create small $\mathrm{C}++$ objects which represent their partial differential equations, boundary conditions, initial conditions, etc. MOOSE will then coordinate PETSc and libMesh to perform a Newton solve over all of the physics to find the solution to the multi-physics problem. While this is still the primary way to use MOOSE, the library has also gained capability for loosely coupled solves, Picard iteration and even coupling to external applications (such as OpenMC and Nek5000). When using MOOSE to couple multiple disparate codes together, a new MOOSE-based application was created which compiles all of the codes into one executable. For the previous study that code was named Cardinal and combines BISON, OpenMC and Nek5000 to achieve high-fidelity simulation of FHRs. 


\subsection{Design of Cardinal}

Cardinal uses the MOOSE MultiApp capability to place each of the applications to be coupled within a hierarchical tree-based structure as shown in Figure 1. This structure was chosen based on how tightly coupled the physics are. BISON and Nek5000 form one branch due to the instantaneous feedback between the conjugate heat transfer and the pebble temperature. The Nek5000 solution provides the temperature boundary condition on the exterior of each pebble while BISON returns the heat flux at each point around the pebble to Nek5000. Another benefit of having BISON and Nek5000 on their own branch is the way it impacts time stepping. Within the MultiApp setup shown in Figure 1 the branch containing BISON and Nek5000 can take many small time steps, and even iterate between BISON and Nek5000 within a time step, without needing to re-solve OpenMC. This greatly increases the runtime speed of the application. OpenMC is then separate from the other two. It receives fuel/pebble temperatures from BISON and returns a heat source which is transferred down to BISON. OpenMC is currently used to solve for steady state neutronics and can therefore take larger time steps compared to BISON and Nek5000 (which are both performing transient heat conduction and CFD solves respectively). The flexibility of the MOOSE MultiApp system allows for just such a setup.

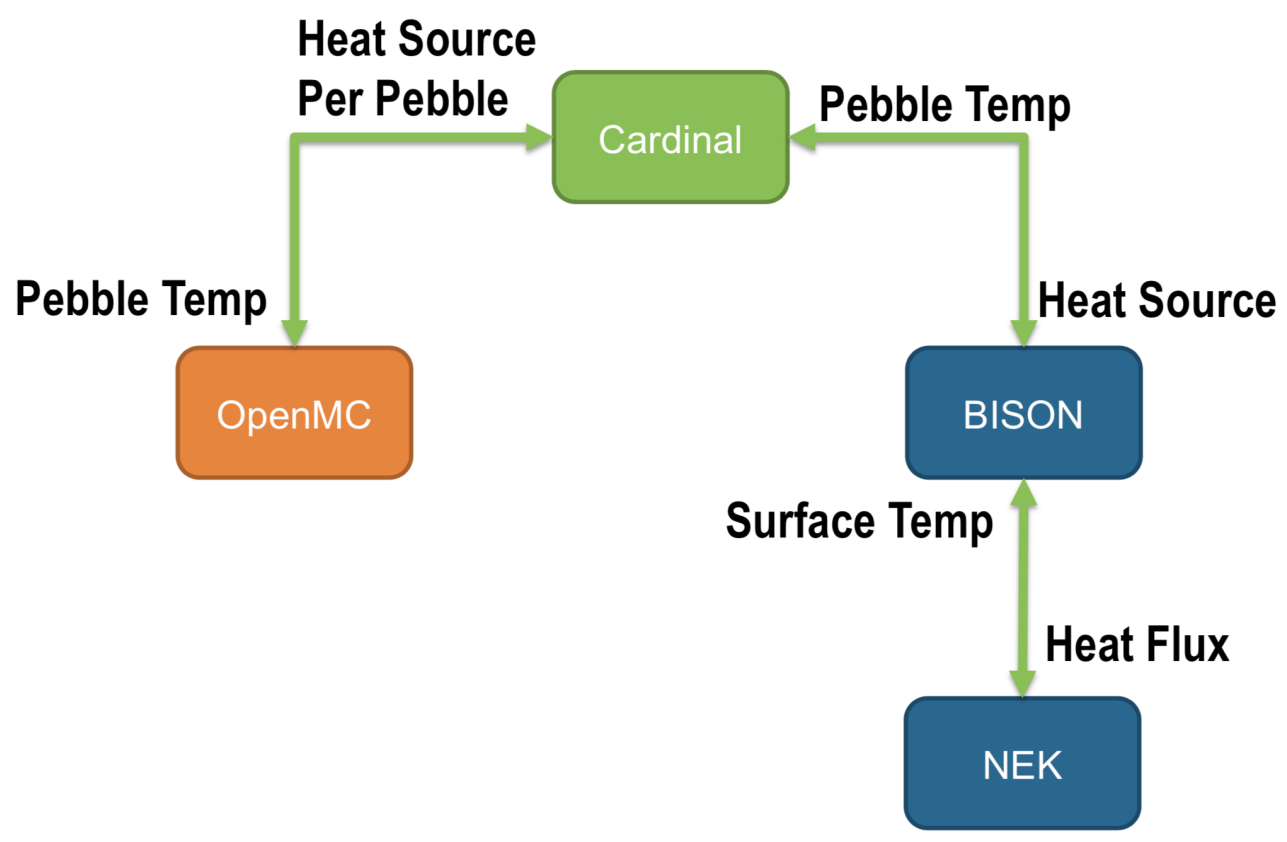

Figure 1: Diagram showing the design of Cardinal.

This structure has been updated in the present report to allow for NekRS to be used instead of Nek5000 allowing for execution of the fluid problem (by far the most expensive of the three) on GPUs. 


\subsection{Build system}

All libraries get configuration info from PETSc for consistent compilation. After installing PETSc and libmesh, Cardinal can be built in one step.

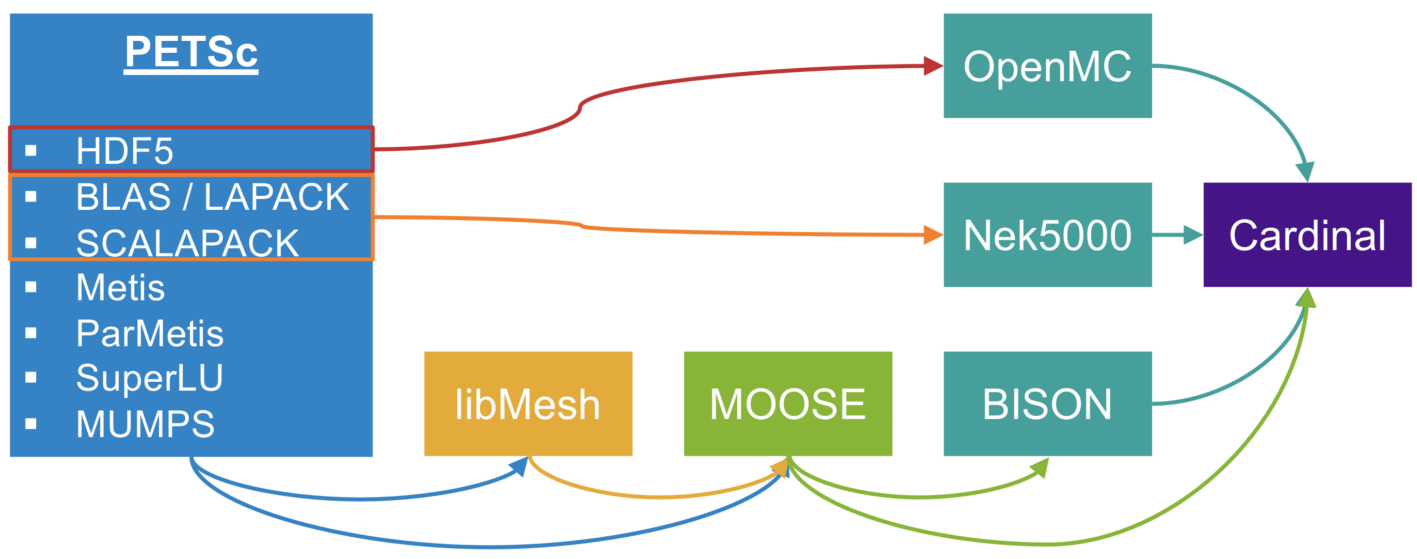

Figure 2: Diagram describing the build system of Cardinal.

The build system of Cardinal has largely been kept intact for the present work. We have however added a branch of the repository that compiles NekRS instead of Nek5000. The build system has also been updated to allow Cardinal to run on the ORNL supercomputer, Summit.

\subsection{Verification and validation}

In order to verify the fluid flow model and the solution transfer we have devised two cases including a single pebble and a two pebble case. The Nek5000-MOOSE coupling was verified to yield the same results as stand-alone Nek5000 conjugate heat transfer results. We note that work in Cardinal was based on previous work conducted on Nek5000-MOOSE coupling [4]. The single pebble (Figure 3) and two pebble (Figure 4) cases were also used to verify the OpenMC and BISON coupling. For instance the neutronics results showed a clear bias between pebbles and a tilt induced by the temperature gradient.

Selected results from the single pebble case were also compared with results from experimental and computational studies carried out by using a similar geometry (Figure 3). Well quantified metrics for flow over a single-sphere are the averaged stream-wise velocity along the domain axial centerline and the azimuthal distribution of the pressure coefficient. Figure 3 compares our result with completed numerical results for the latter. More detailed comparisons for streamwise velocity data can be found in [5]. We do not discuss this quantitatively here, but our result is consistent with the literature.

Over the past several years NEAMS has dedicated several efforts to the modeling and simulation of the detailed flow in a pebble bed. For instance, Fick et al. [6] performed a complete DNS of 

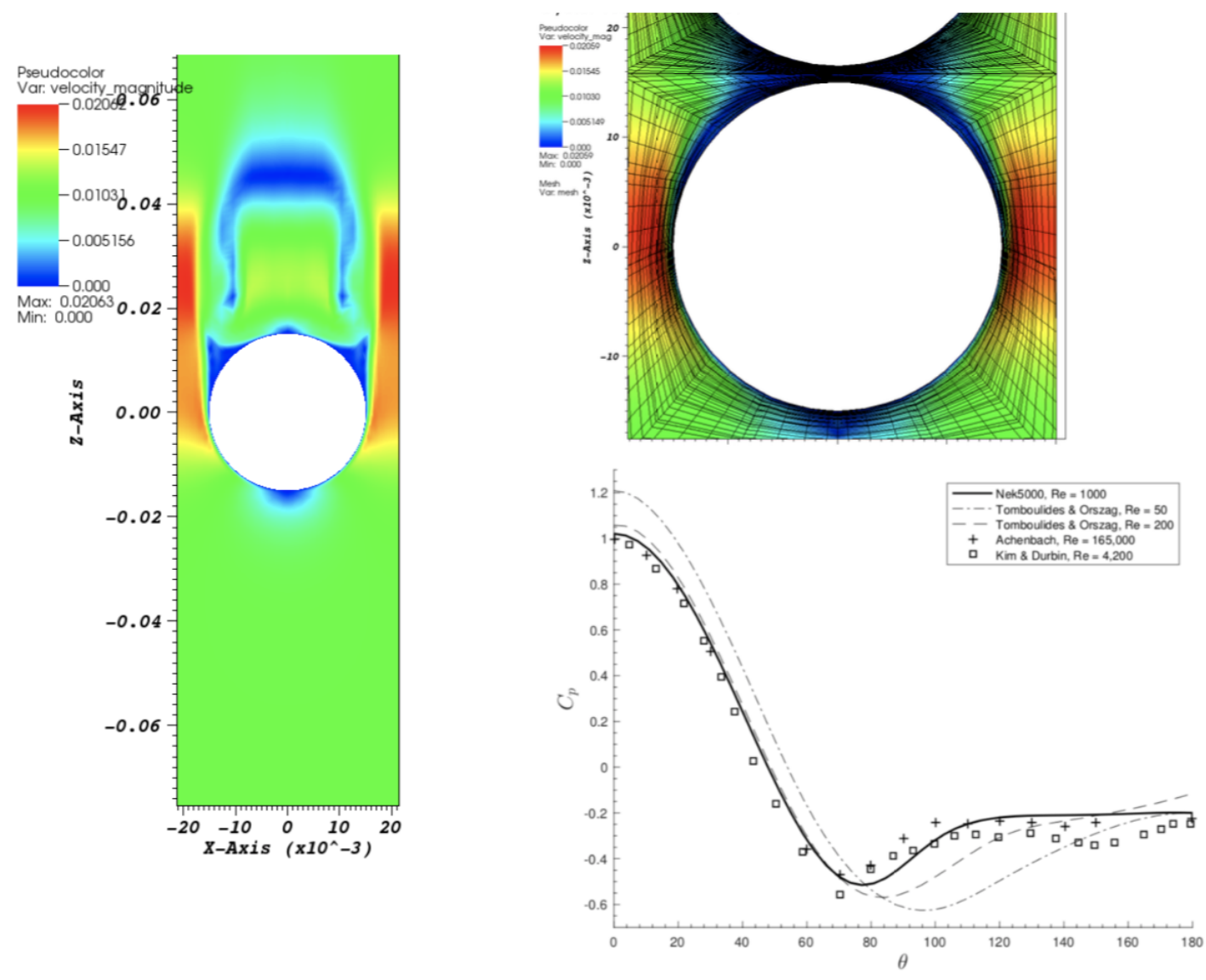

Figure 3: Verification test - Single pebble and comparison with experiment.

pebble bed flow. Complete statistical data was obtained from this DNS study, with an investigation of low-frequency temporal instabilities. However, Fick's study [6] used a structured pebble bed, which limits its application. Nonetheless it was compared against other available DNS data and proved Nek5000 can deliver high quality simulation data for pebble beds. A more recent study was aimed at simulating the flow in a random pebble bed [7]. This random pebble bed geometry was obtained from an experiment conducted by Nguyen et al. [8]. However, only a small section of the whole domain from the experiment was studied. A picture of the experimental facility is shown in Figure 5, while a snapshot of the PIV field examined is shown in Figure 6.

To create a pure hexahedral mesh for a random pebble bed is very challenging if using a traditional blocking method. However, with the tet-to-hex meshing method, a pure hexahedral mesh can be created for this geometry. To reduce the total number of elements, chamfers are created at pebble-pebble interfaces. As discussed, the computational domain is only a small section of the whole experimental domain, therefore we applied periodic boundary conditions at the inlet/outlet to mimic the upstream/downstream. Figure 7 shows the instantaneous velocity field on a cross section of the random pebble bed, as well as the $3 \mathrm{D}$ domain with a section removed to show internal 


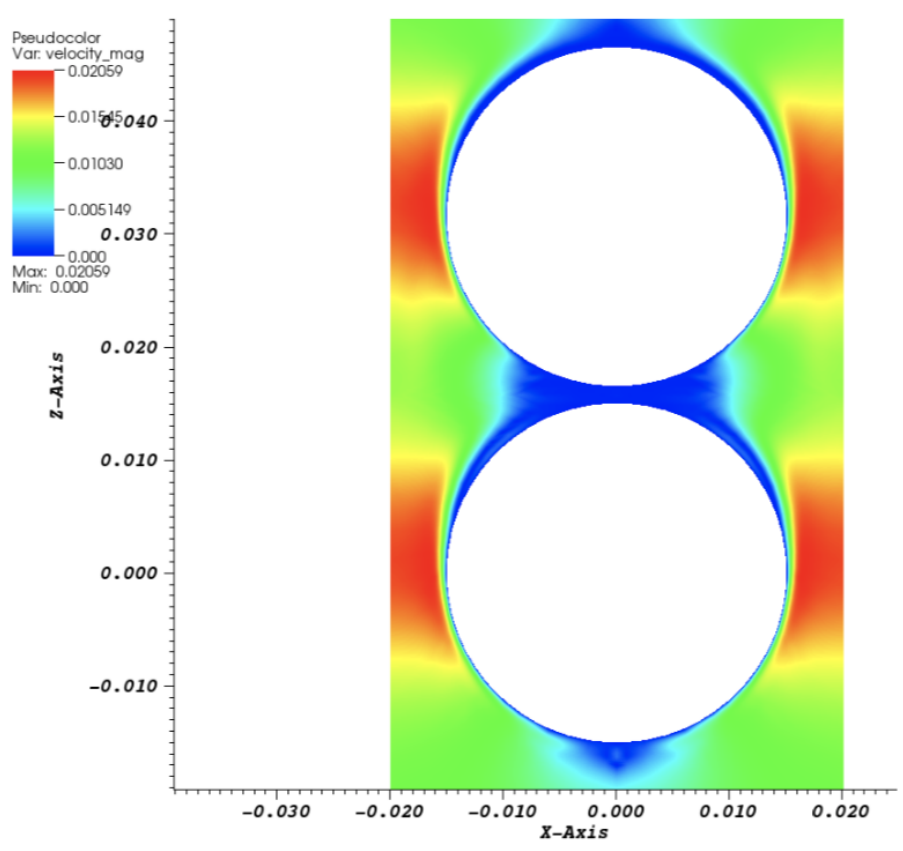

Figure 4: Verification test - Two pebbles.

details. In Figure 8, a direct comparison between DNS results and the experiment is shown. The flow field is very complex due to the randomly distributed pebbles. Despite the complexity of the geometry the computational results compared favorably.

\subsection{Previous coupled simulations}

Using the Nek5000 model of the TAMU experiment as a basis, we have developed a multi-physics simulation of a bed comprising 146 pebbles. We report here only some selected results on the average temperature distribution and power distribution, shown in Figure 9 and Figure 10.

\section{Extension to NekRS}

A good portion of the work on Cardinal this year has been the extension of Cardinal to the use of GPUs. The primary reason for this effort is to exploit the potential of pre-exascale and exascale systems. All such systems in the United States will involve CPU-GPU hybrids. A CFD simulation of a pebble bed reactor core which resolves every pebble requires such computational power. 


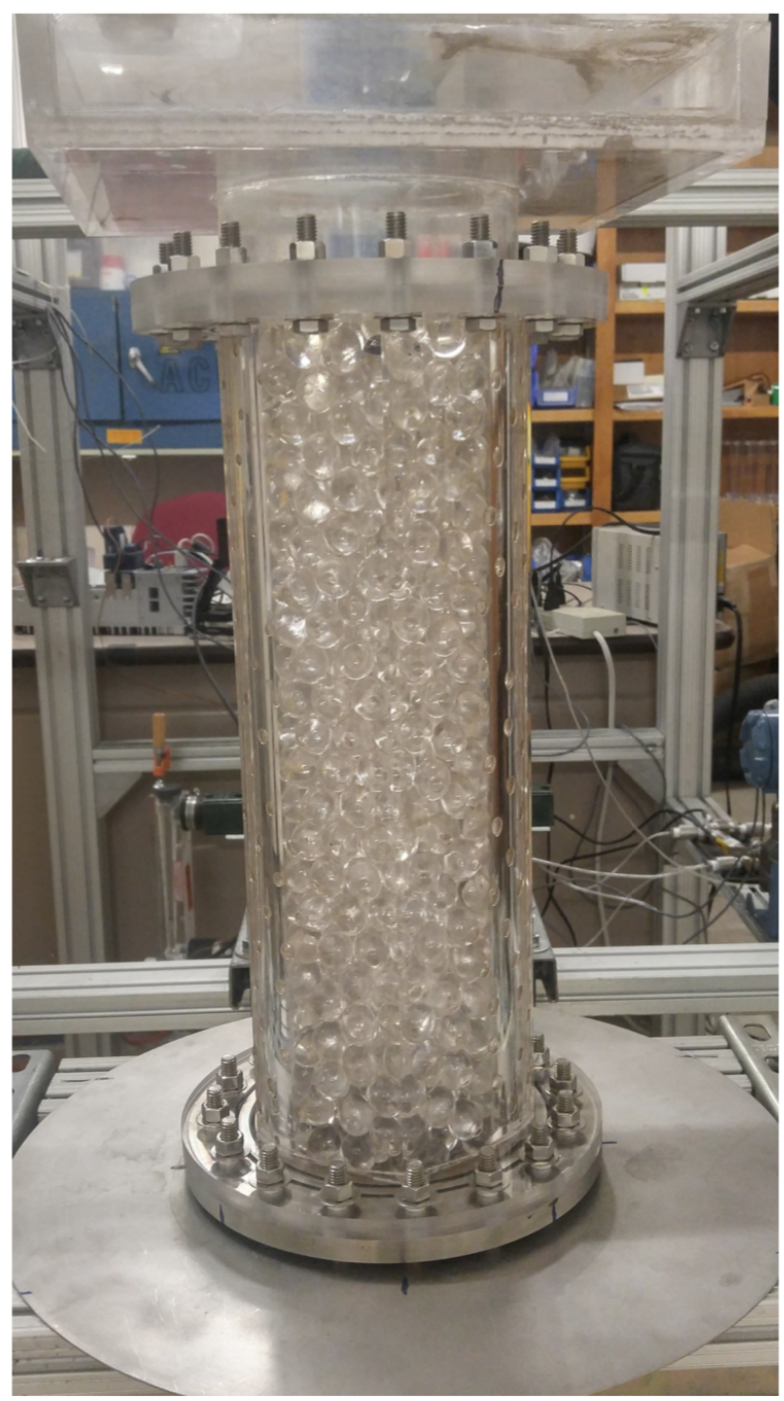

Figure 5: TAMU experiment - Picture of the facility.

\subsection{NekRS}

NekRS is a new GPU-oriented version of Nek5000 that is also capable of running on CPUs. It represents a significant redesign of the code, with major differences. It is primarily written in $\mathrm{C}++$, but it also links to Nek5000 as a library for pre- and post-processing. It has been built primarily under the auspices of the Exascale Computing Program. It is able to reach excellent weak scaling performance. For example, weak-scaling studies performed on Summit at the Oak Ridge Leadership Computing Facility (OLCF), the fastest supercomputer in the world as of June 2020, using NekRS are discussed here. Table 1 shows the solution times, parallel efficiency, and number of points per rank for the Summit results. 


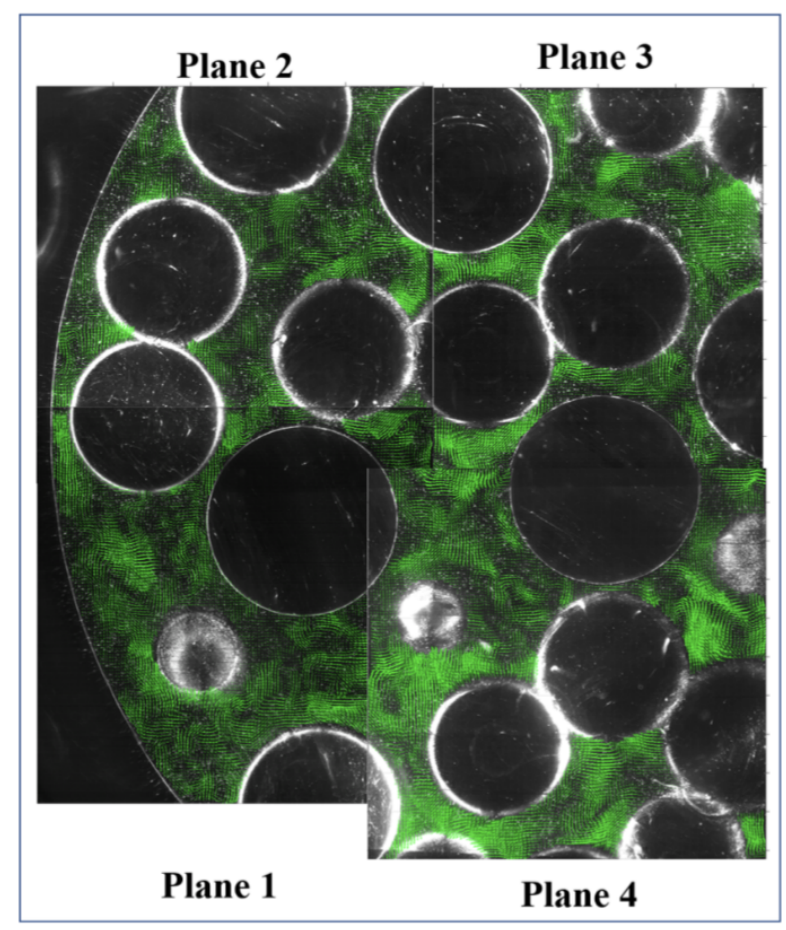

Figure 6: TAMU experiment - PIV snapshot.

We observe in Table 1 near-perfect weak-scaling performance up to 2,048 nodes using 8,000 spectral elements per GPU with a polynomial order $N=7$. The case considered is DNS for a Taylor-Green vortex flow in a triple periodic domain. Timings for 100 time steps were measured. Using six GPUs on 2,048 nodes the runtime was 90 seconds. It is noted that the performance falls off for GPUs when decreasing the degree of freedom (DOF) per GPU. These performance results are in keeping with earlier performance analysis presented in $[9,10]$.

The GPU performance of NekRS is also compared with the CPU performance of Nek5000 using 1,024 nodes on Summit. The CPU simulation was performed with 42 MPI ranks per node and the GPU simulation was performed with 6 GPUs per node. Overall the NekRS GPU solver was $11.5 \times$ faster than the standard Nek5000 CPU solver for the same number of nodes. It is worth noting that NekRS shares a similar verification and validation basis with Nek5000.

Table 1: Weak-scaling on Summit. 8,000 spectral elements per GPU and $N=7$.

\begin{tabular}{ccc}
\hline \# of Nodes on Summit & DOF (billion) & Efficiency on GPUs \\
\hline 128 & 3.1 & 1.0 \\
512 & 12.6 & 0.92 \\
1024 & 25.2 & 0.88 \\
2048 & 50.3 & 0.88 \\
\hline
\end{tabular}



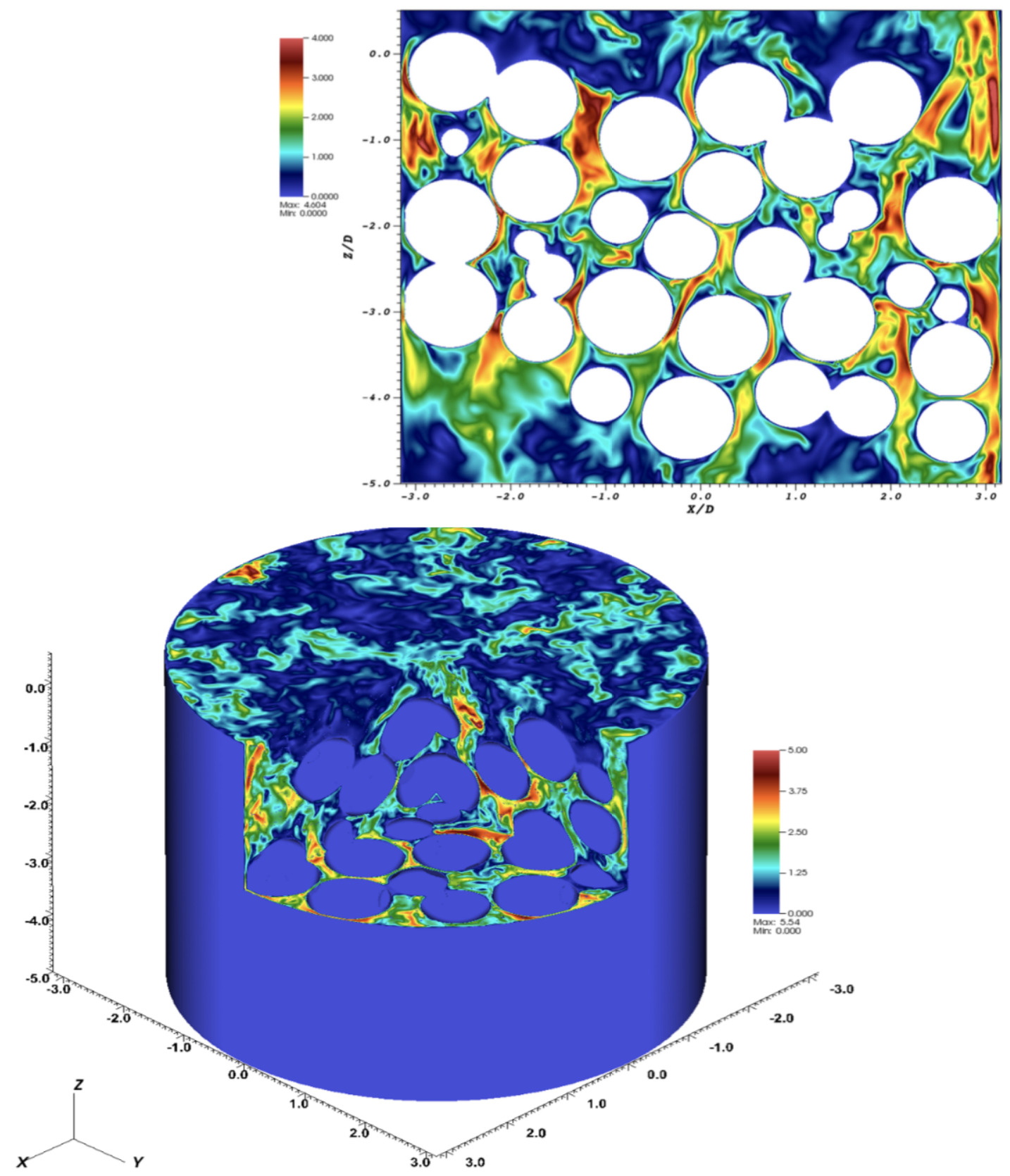

Figure 7: TAMU experiment - Simulation results. 

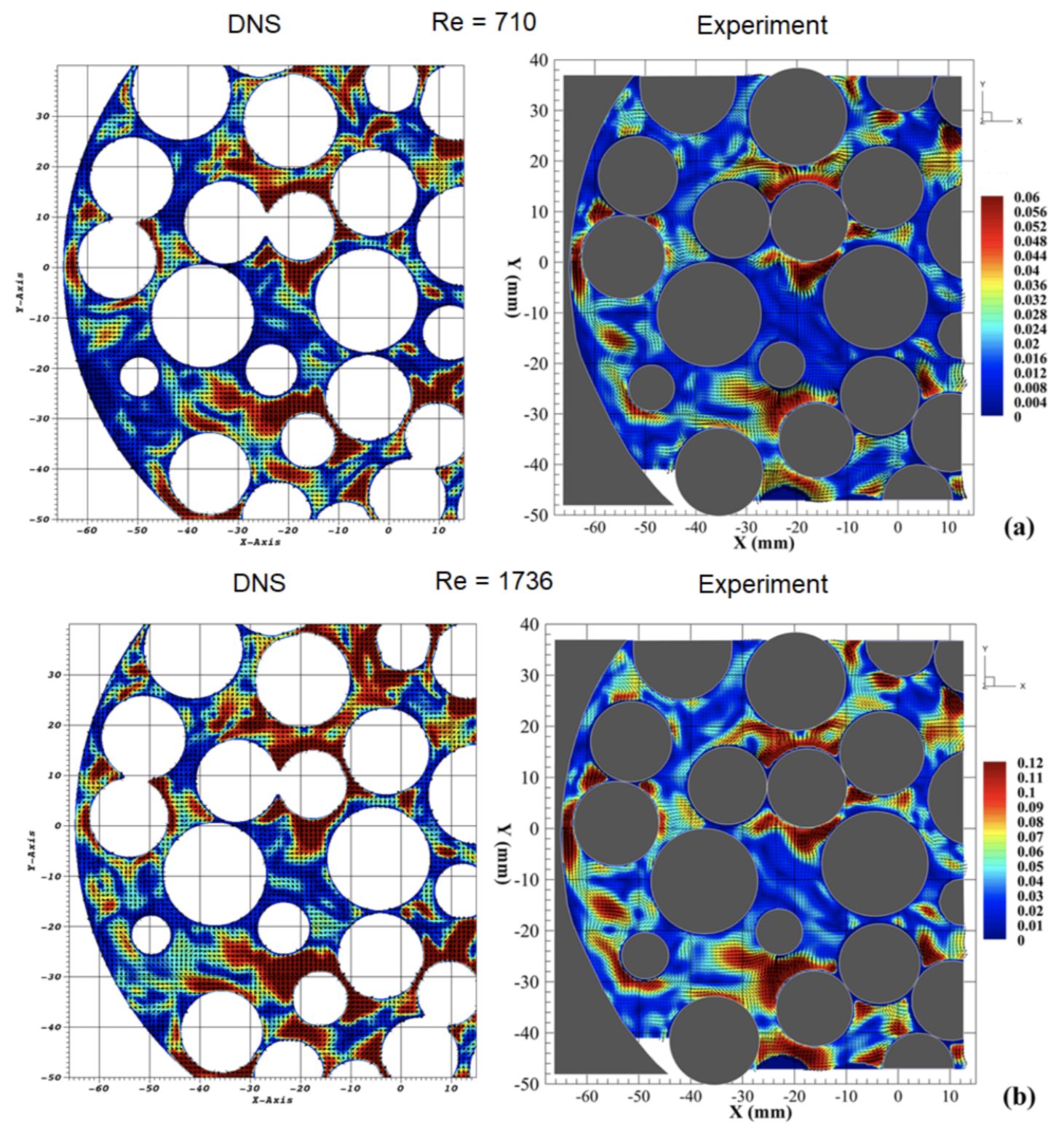

Figure 8: TAMU experiment - Comparison between DNS and the experiment.

\subsection{Updates to Cardinal}

The first step to integrating NekRS into Cardinal has been to create a dedicated branch. This branch has an updated build system that replaces Nek5000 with NekRS. The build system has been tested on various computing systems (Summit, Sawtooth, Workstations on MCS and Macbook laptops) and has been found to be robust.

The second step has been to create an API between NekRS and MOOSE. The interface closely mimics the Nek5000 API and in fact relies on the same Fortran routines for consistency. However, 


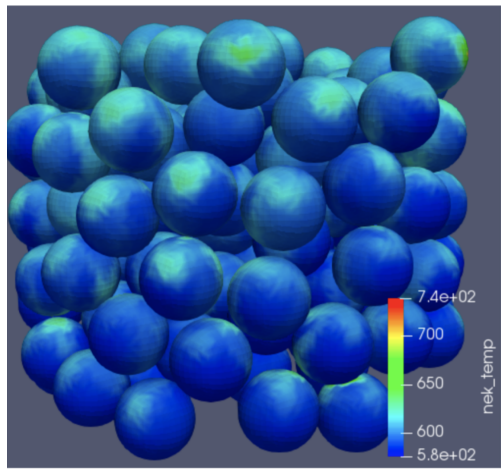

Surface Temperature

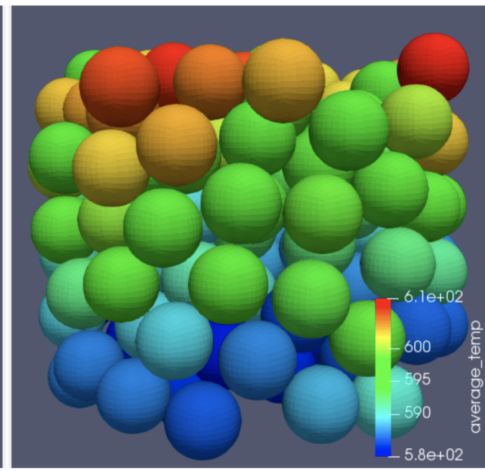

Average Temperature

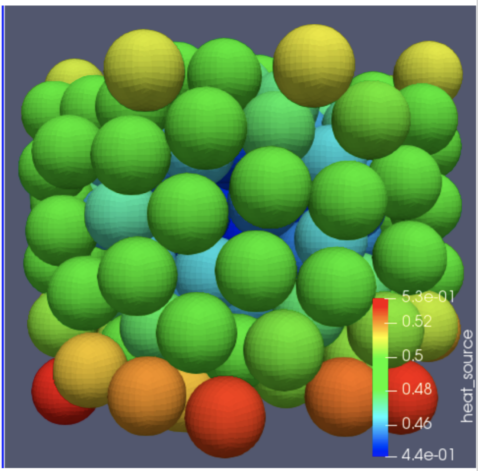

Scaled Heat Source

Figure 9: TAMU demo Results. From left to right: snapshots of temperature on surface, average temperature in solid and average heating
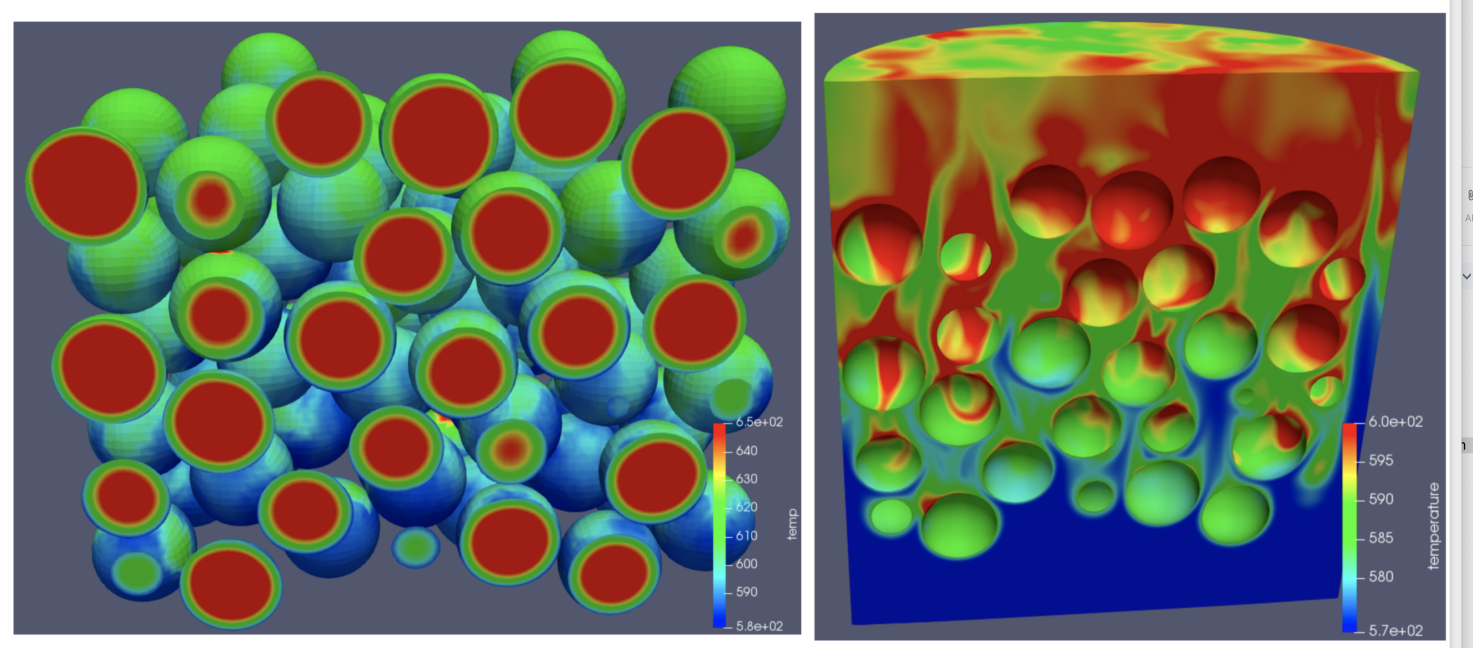

Figure 10: TAMU Demo Results. Right - temperature in the solid. Left - temperature details in the fluid.

additional steps had to be introduced to rely on the very different code structure of NekRS. The updated interface has been tested on the single pebble problem discussed in section 2 . The results of the verification are discussed in subsection 3.3.

The Fortran routines find and list all mesh elements (quadrilateral surface elements) on the surface of the pebble mesh. The quadrilateral data can be linear (built on one quad for each NekRS quad) or quadratic (built on four quads for each NekRS quad). MOOSE then constructs the transfer mesh, which can be distributed or serialized, based on the quadrilateral data. Data for temperature and heat flux are passed between NekRS and MOOSE on the transfer mesh. 


\subsection{Verification}

The results for the NekRS branch of Cardinal are discussed in the following. The case setup is identical to the one used for the verification of the MOOSE-Nek5000 coupling. Some results for the temperature distribution are shown in Figure 11.
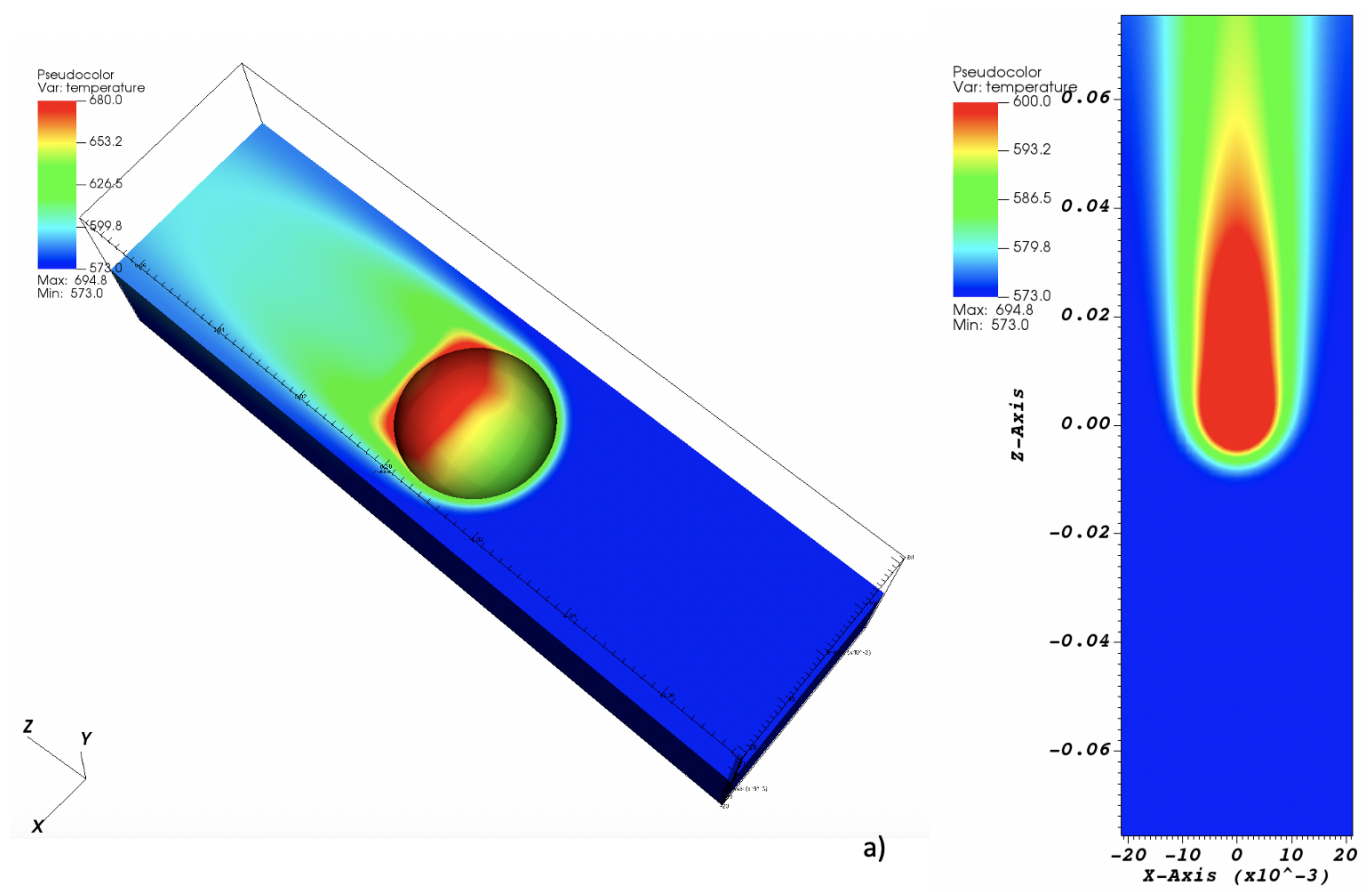

b)

Figure 11: Verification test - Single pebble result. a) 3D temperature distribution in the whole domain. b) Cross section at $y=0.016 \mathrm{~m}$.

Figure 12 presents a comparison between single pebble results in Cardinal versus results obtained in Nek5000 stand-alone with a conjugate heat transfer mesh. Temperature profiles are compared on a line at $y=0.016 \mathrm{~m}$ and $x=0 \mathrm{~m}$ (we note that the domain is centered at the pebble center and the pebble diameter is $D=0.03 m)$. We note that the results are nearly identical if a quadratic representation of the surface is employed.

\section{Major update to OpenMC Heat Source}

In addition to the changes in NekRS some major changes were introduced in the way Cardinal interfaces with OpenMC.

To capture heat generation in the OpenMC simulation, a tally scoring the amount of total recoverable fission energy per source particle is applied to the outer cell of each pebble in the bed. These tallies are created at runtime during the problem setup by using a list of pebble centers to locate the uppermost cell in the CSG geometry hierarchy containing that point. 


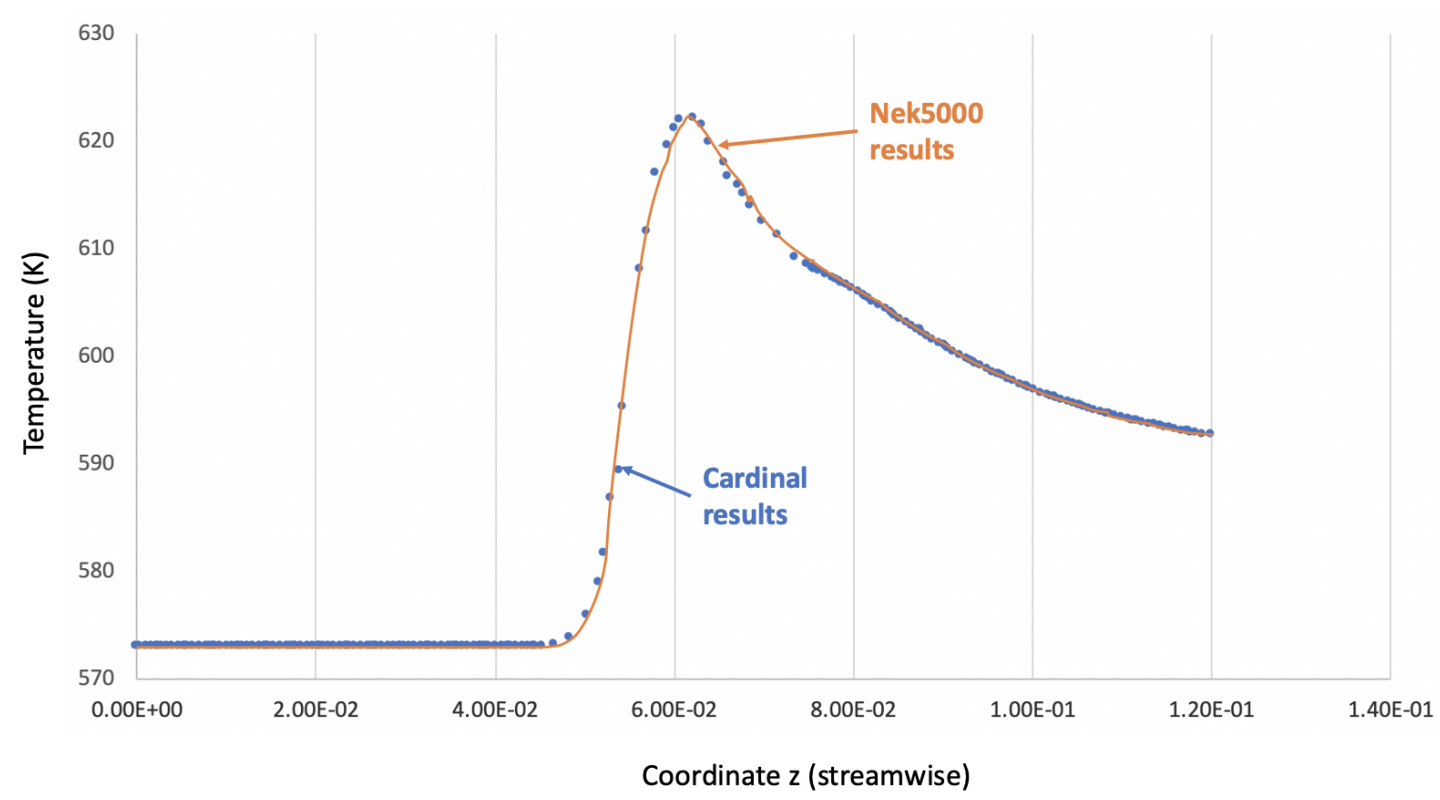

Figure 12: Verification test - Single pebble comparison with standalone Nek5000 results.

As summarized in Equation 1, the energy deposition for each pebble, $\hat{q}_{i}$, is normalized by the total energy deposition in the bed and multiplied by the the total thermal power of the reactor, $Q$ to obtain a an average heat generation rate for each pebble, $q_{i}$. This value is then divided by the volume of each pebble, $V_{p}$, to obtain a volumetric heat generation rate, $q_{i}^{\prime \prime \prime}$.

$$
q_{i}^{\prime \prime \prime}=\frac{Q q_{i}}{V_{p} \sum_{i} \hat{q}_{i}}=\frac{[W][\mathrm{J} / \text { source }]}{\left[\mathrm{cm}^{3}\right][\mathrm{J} / \text { source }]}=\frac{[W]}{\left[\mathrm{cm}^{3}\right]}
$$

This results in a single average heating value for the entire pebble. Before being transferred to the MOOSE mesh, these values are normalized by the total heat

For improved spatial resolution of heat generation in the pebble bed, unstructured mesh tallies have been implemented in OpenMC. Figure 13 provides an example of this capability applied to a single pebble. The unstructured mesh representation relies on a LibMesh mesh instance and currently conforms to the OpenMC model in which the mesh structure and tally data are separated. The separation of this information allows the unstructured mesh to be applied in a mesh filter without knowledge of the underlying mesh type. The mesh filter can then be used in one or more tallies in combination with other specified filters, scores, and nuclides to form a tally. Calls into the LibMesh library from OpenMC are agnostic to the type of elements being used, so meshes can be formed using any element type supported by LibMesh.

A mesh for each pebble is applied in the Cardinal problem to capture the heat generation distribution throughout the pebble bed. To avoid replicating a mesh representing all of the pebbles from either MOOSE or NEKRS, an option for adding translations to mesh filters was introduced in 

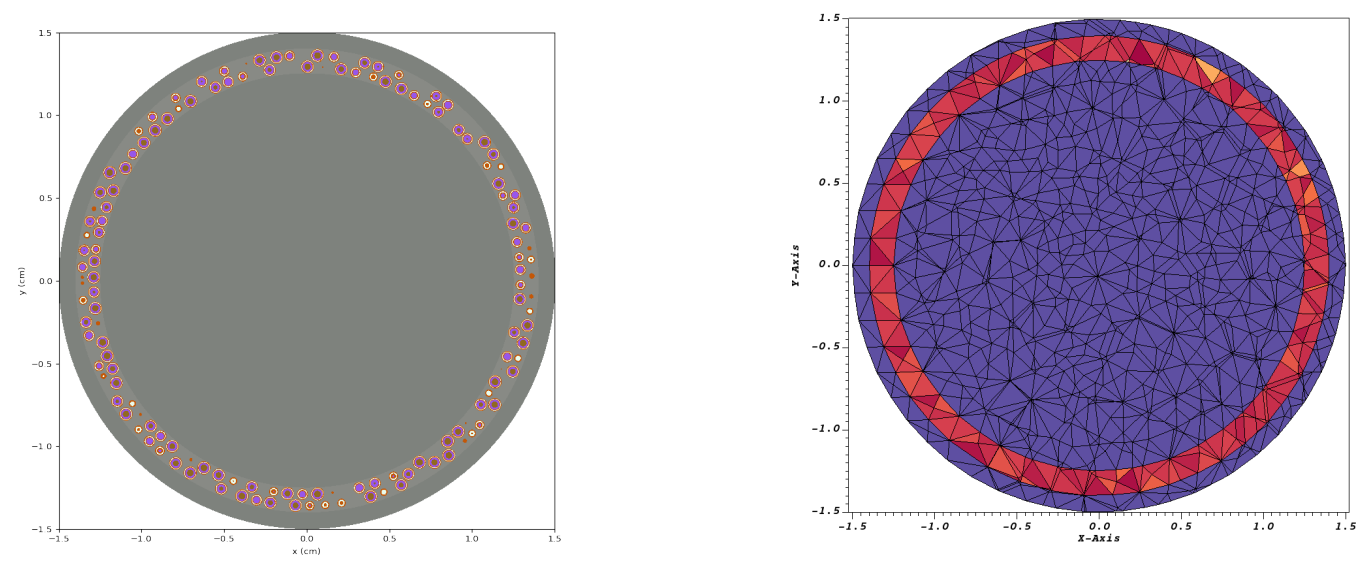

Figure 13: Qualitative view of a heating tally on an unstructured mesh. Left: Cut-away of the pebble geometry as represented in OpenMC. Right: Result of an applied heating tally where heating only occurs in the region of the pebble containing TRISO particles.

OpenMC. Placing the translation at this layer of the tally structure allows all tallies to rely on a single LibMesh mesh instance acting as a template for a single pebble as shown in Figure 14. This results in a very low memory footprint for the tally, even when extended to an entire pebble bed.

The translation values for each mesh filter are set using the list of pebble centers provided in the MOOSE input file. During problem initialization, these translations are used to adjust the location of element centers in the mesh template when transferring heat generation value to MOOSE. As the only additional input information required for the unstructured mesh tallies is the specification of a mesh template input file, the unstructured mesh tallies can be specified at runtime by setting the appropriate tally type parameter in the OpenMC MOOSE input file.

\subsection{Updating Temperature Values}

Temperature values from MOOSE are updated in OpenMC using an average temperature value at the pebble center. To simplify the process of updating the temperature of all cells inside the pebble, an option has been added in OpenMC to propagate a temperature value into all cells filled by a material if the cell whose property is being updated contains a universe or lattice.

\section{Demonstration simulation}

To demonstrate the new capability of Cardinal we demonstrate here the use of Cardinal on Summit to perform coupled multi-physics simulations of pebble beds. 


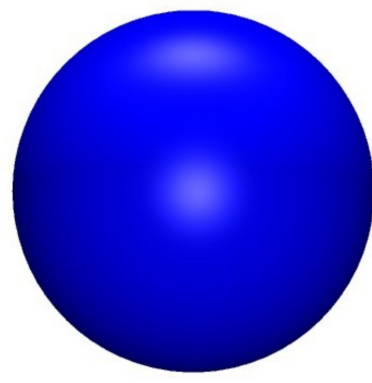

Pebble 1

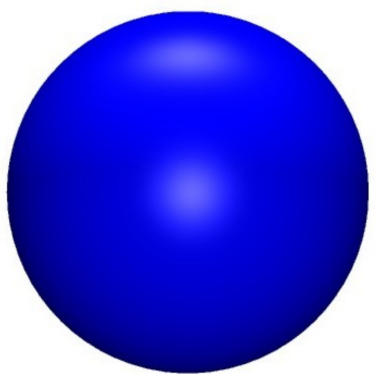

Pebble 2

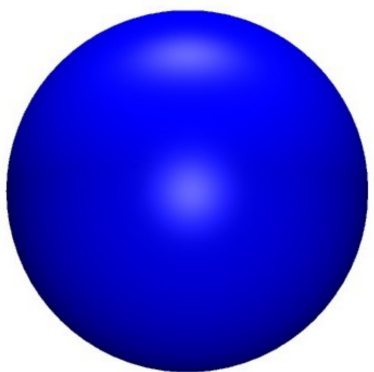

Pebble 3

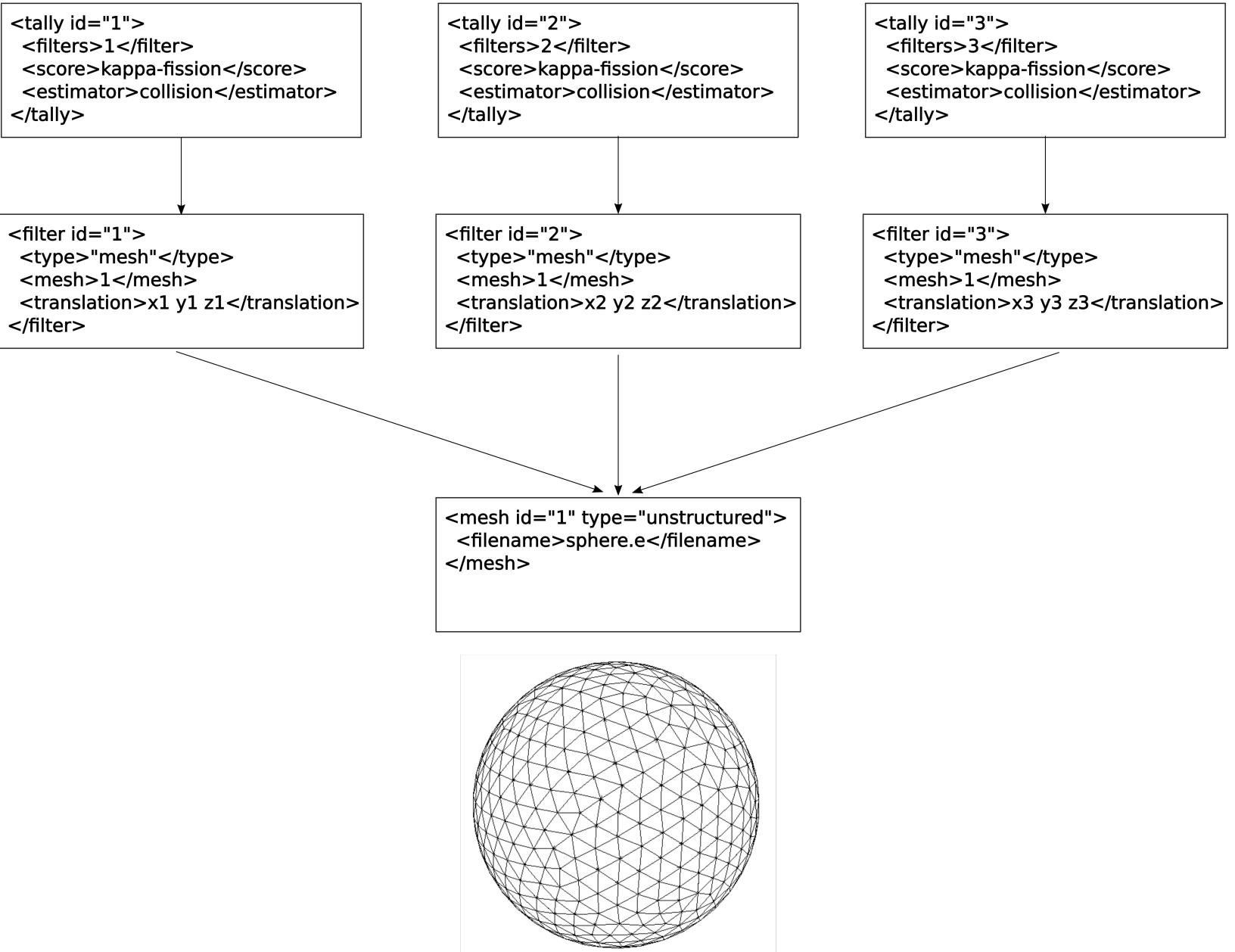

Figure 14: Organization of unstructured mesh tallies for heat generation in OpenMC.

\subsection{Numerical Setup}

The case presented here comprises 1568 pebbles. The pebble configuration has been obtained using a discrete element method (DEM) code [11]. A major overhaul on the mesh generation for the fluid 
domain has been necessary to automate the process as much as possible and reduce the number of elements per pebble. te that NekRS shares a similar verification and validation basis with Nek5000 The new meshing tool is based on a Voronoi cell strategy. It has allowed for the production of high quality hexahedral meshes, while reducing the element count to roughly 300-400 elements per pebble.

Examples for these meshes are provided in Figure 15 for the 1568 pebble configuration and in Figure 16 for a pebble configuration with over 3,000 pebbles. Both meshes have a similar density. We note that 1568 pebbles is a significant size for a coupled calculation and representative for instance of the SANA experiments.

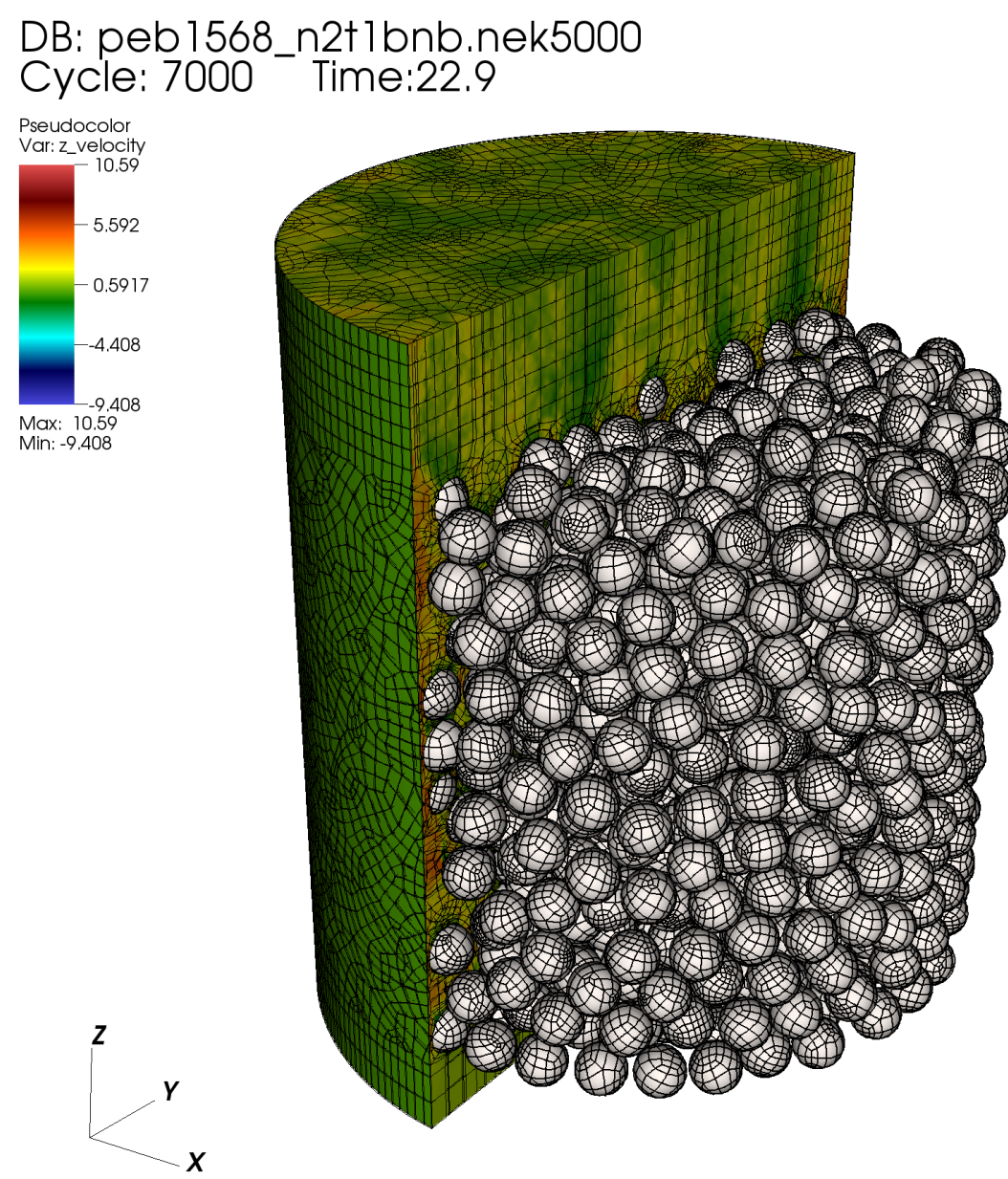

user: ylan

Mon Mar 2 16:25:36 2020

Figure 15: NekRS mesh for 1568 pebble configuration using 524,386 spectral elements.

The principal objective of the new meshing strategy is to reduce the overall element count so that higher polynomial orders (e.g., $N=7$ to 9 ) may be effectively employed. The base elements for the spectral element method are curvilinear hexahedral (hex) elements with an effective resolution of $N^{3}$ points for each of $E$ elements. A minimum value of $n \approx E N^{3}$ is required to capture the 


\section{DB: peb3344_n3.nek5000 \\ Cycle: 9000 Time:24.3}

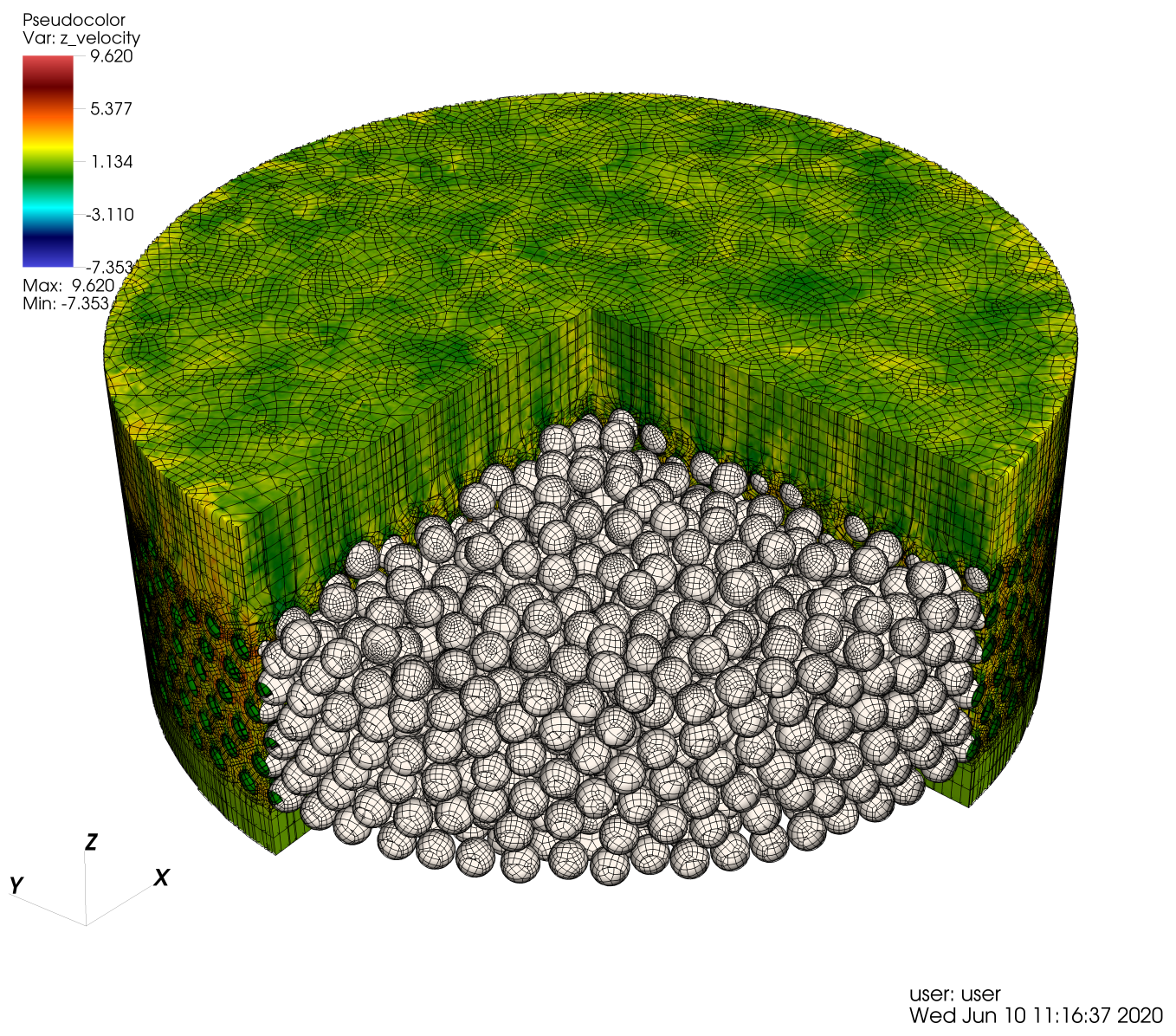

Figure 16: NekRS mesh for 3260 pebble configuration using 1,121,214 spectral elements.

turbulent thermal transport. With a lower element count, $E$, it is possible to elevate the local approximation order. $N=7$ is a nearly optimal value for Nek5000/NekRS because it realizes high throughput with reasonable element counts and reasonable time step sizes [12].

The foundation for the new all-hex meshing strategy is to construct the Voronoi tessellation of the thermal-fluid domain in the void space between the spheres and vessel walls. The Voronoi tessellation has several important properties:

- Each Voronoi cell is convex and closed, with cell walls formed by facets that bisect the line connecting adjacent sphere centers.

- Each facet is a planar convex polygon.

- For $\mathcal{N}$ spheres, Voronoi tessellation has an expected complexity of $O(\mathcal{N})$. 
Each convex polygon can be tessellated into a set of quadrilaterals if we permit insertion of a vertex along edges. For example, if the facet is a triangle, we insert a vertex on each of the three edges and then subdivide the triangle into three quads. Each of these quads can be projected onto the sphere surface to produce a hex element that connects the surface to the Voronoi-cell facet. For a given sphere, one can refine in the radial direction without having the refinement propagate through the domain. The same procedure can be performed for each Voronoi cell (i.e., sphere), so the overall complexity is $O(\mathcal{N})$.

Despite the provably good properties of this approach, a significant concern is that the Voronoi tessellation my still contain very thin facets (i.e., slivers) that lead to poorly conditioned elements. To overcome this and to generate a more uniform mesh that has a bounded ratio of longest to shortest edge we first perform edge collapse on the Voronoi cells. Any edge that is shorter than a given tolerance is collapsed and its two vertices are fused into one. If, after edge collapse, the number of edges on a facet is $<3$, the facet is deleted. Subsequent to edge collapse, we use vertex insertion to ensure that the longest edge is below a certain threshold. The risk of edge collapse is that facets lose their planarity and, worse, might not actually face the sphere that they are nominally bounding. These situations require some care to recover to ensure that the resulting all-hex mesh has valid positive Jacobians for each element.

Once the mesh is constructed, it is smoothed using a combination of Laplacian smoothing and element-Jacobian optimization where the objective function includes a substantial penalty for negative Jacobians.

For these demonstrations, the sizes and composition of the TRISO particles were based on TRISO manufactured at INL.Though these particles were developed for the Advanced Gas Reactor (AGR) fuel, particles with the same specifications are used for FHR test reactors and computation benchmarks. The sizes and compositions of the pebbles were taken from the Mk1 PB-FHR reactor constructed at UC Berkeley.

For BISON, and the demonstration problem under consideration, we consider only the conduction equation and as such it is a relatively straightforward setup. Properties are constant and adapted from available correlations. The mesh for a single sphere is generated and replicated at run time. The same mesh is used for the mesh tallies in OpenMC.

\subsection{Results}

The model described in the previous section has been run on 12 and 20 nodes of Summit, with 6 MPI ranks on each node, corresponding to the 6 GPUs on each node. The OpenMC and BISON models are designed to run on the CPU while the NekRS model runs on the GPU.

Stand-alone NekRS simulations have been run first up to 25 convective time units to develop turbulence - Figure 17 using an LES approach. A restart file has then been generated and used to restart a transient simulation in Cardinal representing a heat-up of the pebbles. The time step has been fixed to $5\left(10^{-4}\right) \mathrm{s}$ in both BISON and NekRS. The temperature at time zero has been set to $300^{\circ} \mathrm{C}$ everywhere. Figure 18 presents the temperature at the surface of the pebbles in BISON 


\section{DB: peb1568_n2†1bnb.nek5000 \\ Cycle: 7000 Time:22.9}

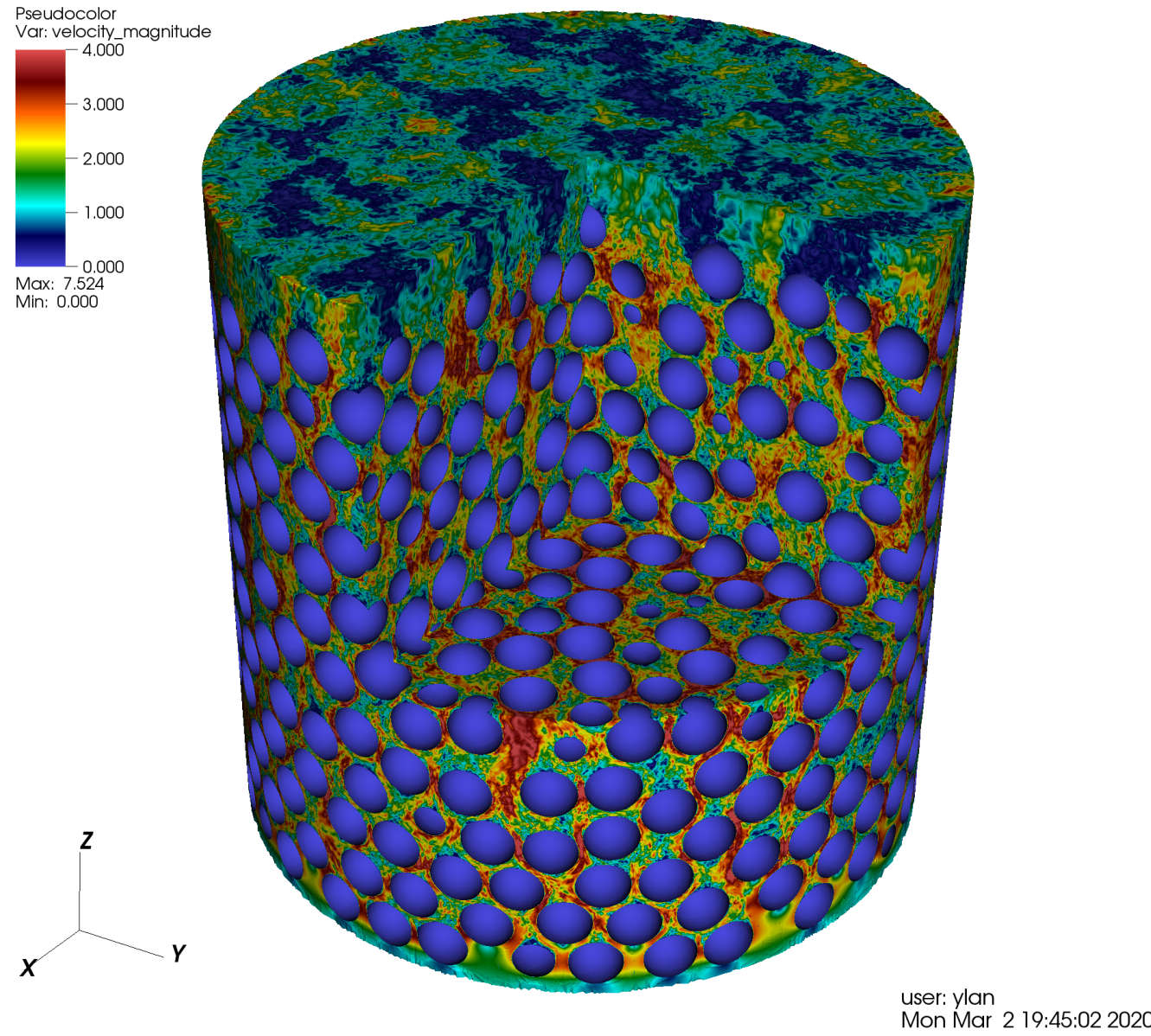

Figure 17: NekRS results for the velocity field.

at three points in time. The simulations took $2.5 \mathrm{~s}$ per coupled time step on 20 nodes, requiring transfer between physics at each time step. However, this could be greatly optimized by relaxing the requirement, as data transfer from GPU to CPU should be minimized as much as possible.

The results of OpenMC simulations coupled with the heat conduction module are shown in Figure 19 and Figure 20 with the same time step parameters as the NekRS simulations described above. An eigenvalue simulation using 150 batches with 50 inactive batches was executed in each time step. 50,000 particles per batch were used to converge the pebble-averaged heat source from the OpenMC cell tally while the unstructured mesh heat source tally required 500,000 particles per batch to produce the heat source presented here. Production simulations may require an even higher number of particles per batch to more tightly converge the heating distribution when using the unstructured mesh heat source due to the decreased number of samples per source particle in the tally bins. Figure 21 demonstrates the effect of the improved spatial resolution provided by the unstructured mesh heat source from OpenMC on the temperature distribution within a 

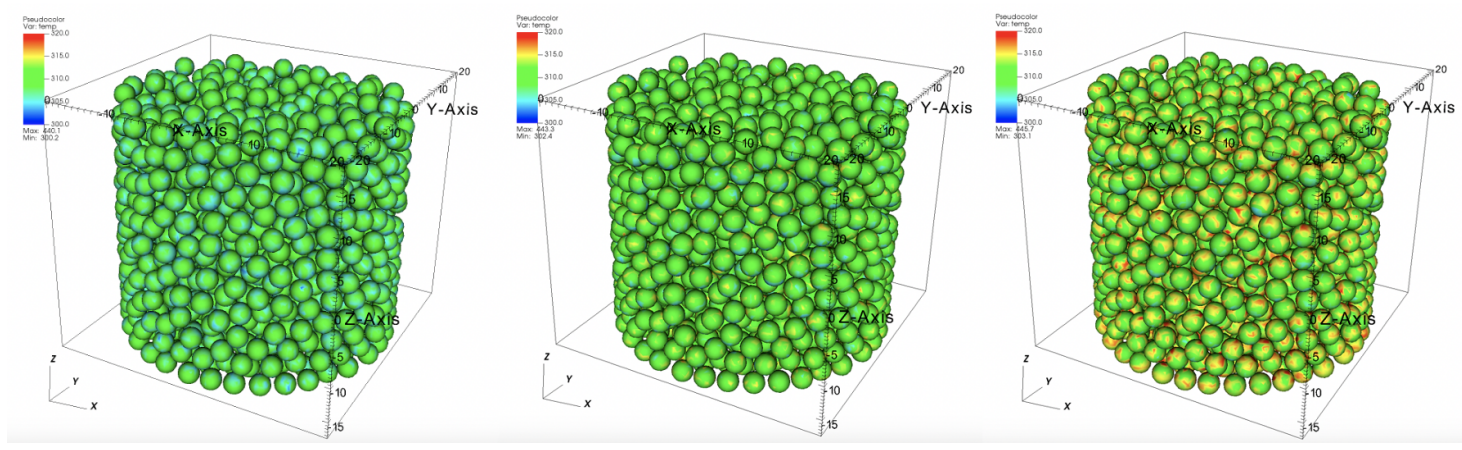

Figure 18: Temperature result for the pebble surface temperature at three points in time.
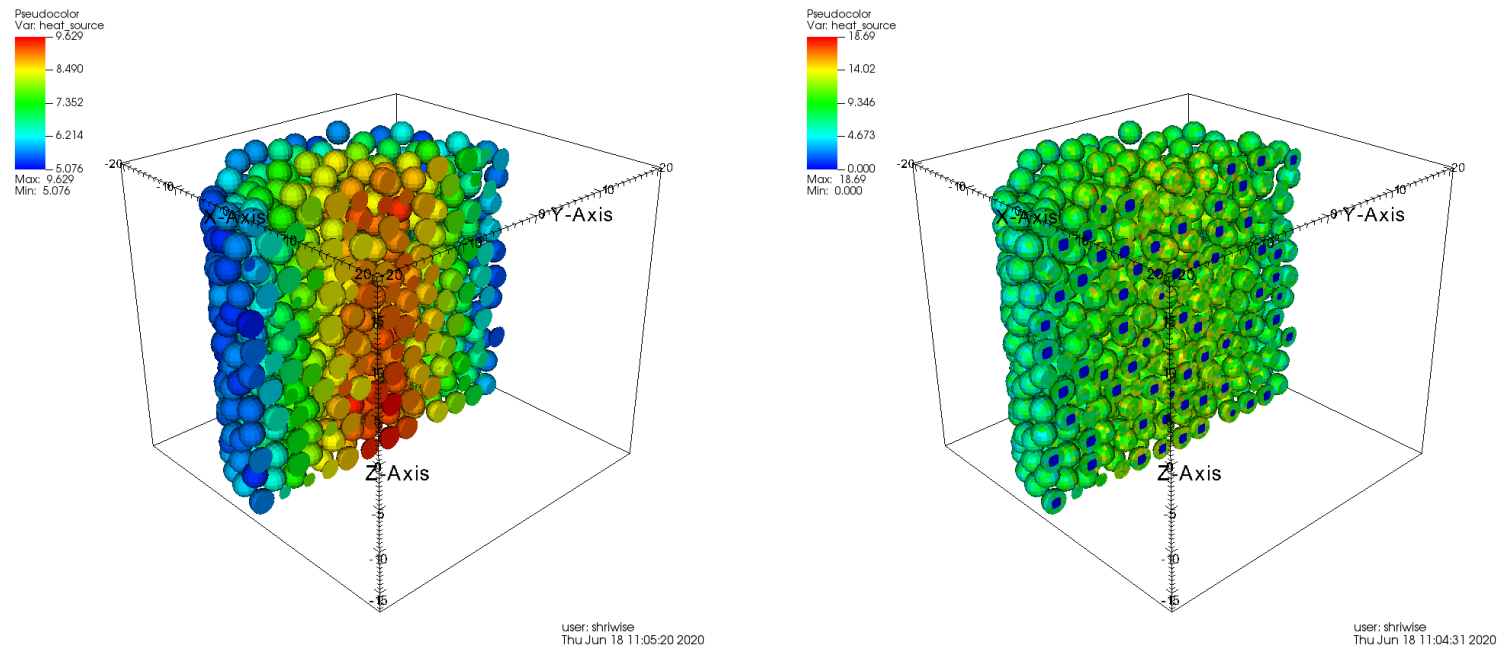

Figure 19: Left: Heat source using the original cell tallies to produces an average heat source per-pebble. Right: Heat source produced using an OpenMC unstructured mesh tally.

representative pebble. In the case of the pebble-averaged heat source the temperature profile is symmetric. This reflects the uniform heat source applied in that region. An asymmetry can be seen in the profile generated from the unstructured mesh heat source, indicating that the improved spatial resolution of the source has an impact on the temperature distribution in the solid. This will in turn affect the resulting heat transfer to the fluid.

\subsection{Projection to full core}

20 nodes of Summit represents less than $1 \%$ of the computing power available on Summit. We estimate that $80 \%$ of the machine will be sufficient to perform full core calculations in FHRs corresponding to 300,000 pebbles. 

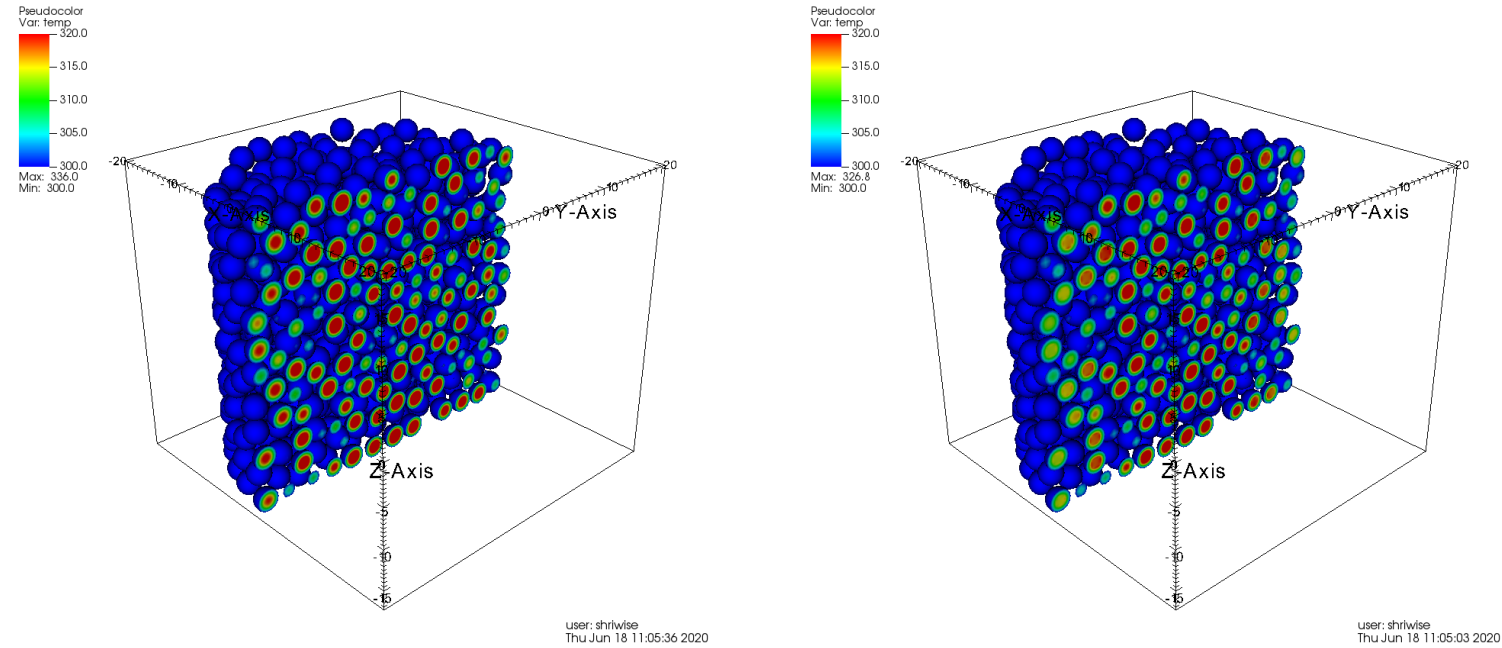

Figure 20: Left: Temperature in the solid resulting from the cell-based heating tally. Right: Temperature in the solid resulting from the unstructured mesh heating tally in OpenMC.
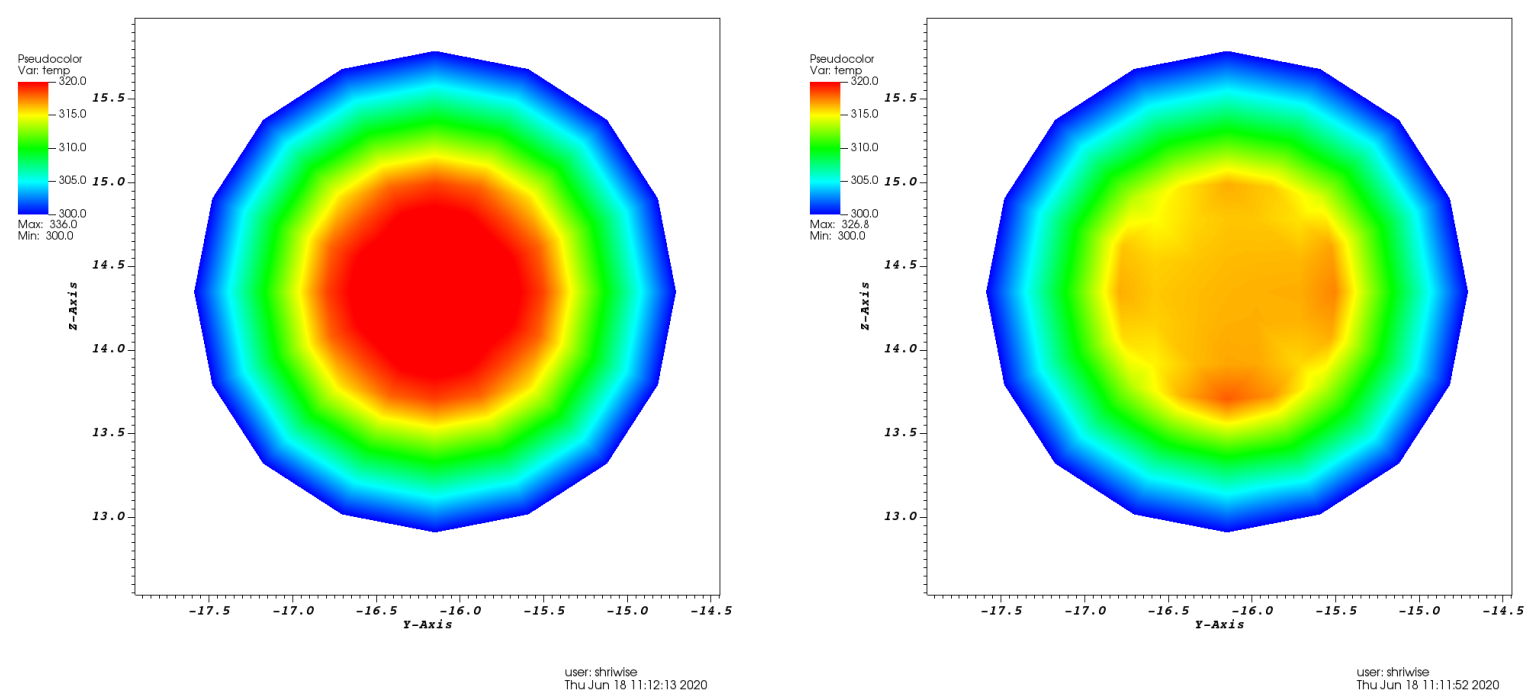

Figure 21: Temperature profiles of the same pebble in the 1568 pebble demo using the pebbleaveraged heating (left) and the unstructured mesh heating (right).

\section{Conclusions and Future Work}

In this report we describe the extension of Cardinal to GPUs including a demonstration simulation on Summit. Cardinal provides a platform for running coupled OpenMC+(Nek5000/NekRS $)+B I S O N$. We demonstrated the application of Cardinal with a first-of-a kind simulation on Summit, representing a $10 \times$ pebble count increase compared to previous simulations. This exercise demonstrates that the 
MOOSE-Wrapped-App paradigm is working well even on a GPU platform like Summit.

We aim to conduct massively parallel simulations going up to $80 \%$ of Summit for full core simulations. The current results provide a clear pathway toward full core simulations. Future work on Cardinal will involve a more sophisticated thermal contact treatment between pebbles, leveraging novel ideas using MOOSE constraints. Radiation heat transfer will also be added.

These simulations may involve asynchronous parallel execution between the physics. Finally a multi-scale approach may be employed to simulate a select number of fuel particles directly.

\section{Acknowledgments}

Argonne National Laboratory's work was supported by the U.S. Department of Energy, Office of Nuclear Energy, Nuclear Energy Advanced Modeling and Simulation (NEAMS), under contract DE-AC02-06CH11357. 


\section{References}

[1] A. T. Cisneros, J. K. Choi, A. Y. Chong, M. Fratoni, and S. Hong, "Technical description of the mark 1 pebble-bed fluoride-salt-cooled high-temperature reactor (pb-fhr) power plant," in Tech. Rep., Department of Nuclear Engineering, University of California Berkeley, 2014.

[2] D. Gaston, C. Newman, G. Hansen, and D. Lebrun-Grandie, "Moose: A parallel computational framework for coupled systems of nonlinear equations," Nuclear Engineering and Design, vol. 239, no. 10, pp. 1768-1778, 2009.

[3] E. Merzari, "Toward exascale: Large eddy simulation and direct numerical simulation of nuclear reactor flows with the spectral element method," in 18th International Topical Meeting on Nuclear Reactor Thermal Hydraulics, NURETH 2019, 2019.

[4] A. Novak, P. Romano, B. Wendt, R. Rahaman, E. Merzari, L. Kerby, C. Permann, R. Martineau, and R. Slaybaugh, "Preliminary coupling of openmc and nek5000 within the moose framework," in Proceedings of PHYSOR, 2018.

[5] L. H. Fick, E. Merzari, O. Marin, and Y. A. Hassan, "Investigation of the dynamics of incompressible flow in domains of multiple close-packed spheres," in ASME 2017 Fluids Engineering Division Summer Meeting, American Society of Mechanical Engineers Digital Collection, 2017.

[6] L. H. Fick, E. Merzari, and Y. A. Hassan, "Direct numerical simulation of pebble bed flows: Database development and investigation of low-frequency temporal instabilities," Journal of Fluids Engineering, vol. 139, no. 5, 2017.

[7] M. A. Yildiz, G. Botha, H. Yuan, E. Merzari, R. C. Kurwitz, and Y. A. Hassan, "Direct numerical simulation of the flow through a randomly packed pebble bed," Journal of Fluids Engineering, vol. 142, no. 4, 2020.

[8] T. Nguyen, E. Kappes, S. King, Y. Hassan, and V. Ugaz, "Time-resolved piv measurements in a low-aspect ratio facility of randomly packed spheres and flow analysis using modal decomposition," Experiments in Fluids, vol. 59, no. 8, p. 127, 2018.

[9] P. Fischer, K. Heisey, and M. Min, "Scaling limits for PDE-based simulation (invited)," in 22nd AIAA Computational Fluid Dynamics Conference, AIAA Aviation, AIAA 2015-3049, 2015. 
[10] M. Otten, J. Gong, A. Mametjanov, A. Vose, J. Levesque, P. Fischer, and M. Min, "An MPI/OpenACC implementation of a high order electromagnetics solver with GPUDirect communication," Int. J. High Perf. Comput. Appl., 2016.

[11] Project Chrono, "Chrono: An Open Source Framework for the Physics-Based Simulation of Dynamic Systems." http://projectchrono.org. Accessed: 2016-03-07.

[12] P. Fischer, M. Min, T. Rathnayake, S. Dutta, T. Kolev, V. Dobrev, J.-S. Camier, M. Kronbichler, T. Warburton, K. Swirydowicz, and J. Brown, "Scalability of high-performance PDE solvers," IJHPCA, vol. in press, 2020. 


\section{Argonne}

Nuclear Science and Engineering Division

Argonne National Laboratory

9700 South Cass Avenue, Bldg. 208

Argonne, IL 60439

www.anl.gov 